\title{
The complex spin state of 103P/Hartley 2: Kinematics and orientation in space
}

\author{
Michael J.S. Belton ${ }^{\mathrm{a}, *}$, Peter Thomas ${ }^{\mathrm{b}}$, Jian-Yang Li ${ }^{\mathrm{c}}$, Jade Williams ${ }^{\mathrm{c}}$, Brian Carcich ${ }^{\mathrm{b}}$, Michael F. A'Hearn ${ }^{\mathrm{c}}$, \\ Stephanie McLaughlin ${ }^{c}$, Tony Farnham ${ }^{c}$, Lucy McFadden ${ }^{\mathrm{d}}$, Carey M. Lisse ${ }^{\mathrm{e}}$, Steven Collins ${ }^{\mathrm{f}}$, \\ Sebastien Besse ${ }^{c}$, Kenneth Klaasen ${ }^{f}$, Jessica Sunshine ${ }^{c}$, Karen J. Meech ${ }^{g}$, Don Lindler ${ }^{\mathrm{h}}$ \\ ${ }^{a}$ Belton Space Exploration Initiatives, LLC, 430 S. Randolph Way, Tucson, AZ 85716, USA \\ ${ }^{\mathrm{b}}$ Center for Radiophysics and Space Research, Cornell University, Ithaca, NY 14853, USA \\ ${ }^{\mathrm{c}}$ Department of Astronomy, University of Maryland, College Park, MD 20742, USA \\ ${ }^{\mathrm{d}}$ NASA/Goddard Space Flight Center, Greenbelt, MD 20771, USA \\ ${ }^{\mathrm{e}}$ Planetary Exploration Group, Space Department, Johns Hopkins University Applied Physics Laboratory, 11100 Johns Hopkins Rd., Laurel, MD 20723, USA \\ f Jet Propulsion Laboratory, California Institute of Technology, 4800 Oak Grove Dr., Pasadena, CA 91109, USA \\ ${ }^{\mathrm{g}}$ Institute for Astronomy, University of Hawaii, Honolulu, HI 96822, USA \\ ${ }^{\mathrm{h}}$ Sigma Space Corporation, Lanham, MD 20706, USA
}

\section{A R T I C L E I N F O}

\section{Article history:}

Available online 16 July 2012

\section{Keywords:}

Comets

Comets, Nucleus

Rotational dynamics

\begin{abstract}
A B S T R A C T
We derive the spin state of the nucleus of Comet 103P/Hartley 2, its orientation in space, and its shortterm temporal evolution from a mixture of observations taken from the DIXI (Deep Impact Extended Investigation) spacecraft and radar observations. The nucleus is found to spin in an excited long-axis mode (LAM) with its rotational angular momentum per unit mass, $\mathbf{M}$, and rotational energy per unit mass, $E$, slowly decreasing while the degree of excitation in the spin increases through perihelion passage. $\mathbf{M}$ is directed toward (RA, Dec; J2000) $=8 \pm 4^{\circ}, 54 \pm 1^{\circ}$ (obliquity $=48 \pm 1^{\circ}$ ). This direction is likely changing, but the change is probably $<6^{\circ}$ on the sky over the $\sim 81.6$ days of the DIXI encounter. The magnitudes of $\mathbf{M}$ and $E$ at closest approach (JD 2455505.0831866 2011-11-04 13:59:47.310) are $30.0 \pm 0.2 \mathrm{~m}^{2} / \mathrm{s}$ and $(1.56 \pm 0.02) \times 10^{-3} \mathrm{~m}^{2} / \mathrm{s}^{2}$ respectively. The period of rotation about the instantaneous spin vector, which points in the direction (RA, Dec; J2000) $=300 \pm 3.2^{\circ}, 67 \pm 1.3^{\circ}$ at the time of closest approach, was $14.1 \pm 0.3 \mathrm{~h}$. The instantaneous spin vector circulates around $\mathbf{M}$, inclined at an average angle of $33.2 \pm 1.3^{\circ}$, with an average period of $18.40 \pm 0.13 \mathrm{~h}$ at the time of closest approach. The period of roll around the principal axis of minimum inertia ("long" axis) at that time is $26.72 \pm 0.06 \mathrm{~h}$. The long axis is inclined to $\mathbf{M}$ by $\sim 81.2 \pm 0.6^{\circ}$ on average, slowly decreasing through encounter. We infer that there is a periodic nodding motion of the long axis with half the roll period, i.e., $13.36 \pm 0.03 \mathrm{~h}$, with amplitude of $\sim 1^{\circ}$ again decreasing through encounter. The periodic variability in the circulation and roll rates during a cycle was at the $2 \%$ and $10-14 \%$ level respectively.

During the encounter there was a secular lengthening of the circulation period of the long axis by $1.3 \pm 0.2 \mathrm{~min} / \mathrm{d}$, in agreement with ground-based estimates, while the period of roll around the long axis changed by $\sim-4.4 \mathrm{~min} / \mathrm{d}$ at perihelion. $\mathbf{M}$ decreased at a rate of $-0.038\left(\mathrm{~m}^{2} / \mathrm{s}\right)$ per day in a roughly linear fashion. Assuming a bulk density between $230-300 \mathrm{~kg} / \mathrm{m}^{3}$ and a total volume for the nucleus of $8.09 \times 10^{8} \mathrm{~m}^{3}$, the net torque acting on the nucleus was in the range $0.8-1.1 \times 10^{5} \mathrm{~kg} \mathrm{~m}^{2} / \mathrm{s}^{2}$. In order to bring the spacecraft photometric and imaging data into alignment on the direction of $\mathbf{M}$, the directions of the intermediate and short principal axes of inertia had to be adjusted by $33^{\circ}$ (on the sky) from the values indicated by the shape model with an assumed homogeneous interior. The adjusted direction of the intermediate axis is RA, Dec $=302^{\circ},-16.5^{\circ}$. The morning and evening terminators in the images are identified, and the variation of the insolation at three regions on the nucleus associated with active areas calculated. The plume of water vapor observed in the inner coma is found to be directed close to the direction of local gravity over the sub-solar region for a range of reasonable bulk densities. The plume does not follow the projected normal to the surface at the sub-solar point.
\end{abstract}

() 2012 Elsevier Inc. All rights reserved.

\footnotetext{
* Corresponding author. Fax: +1 5207956286.

E-mail address: mbelton@dakotacom.net (M.J.S. Belton).
}

\section{Introduction}

Prior to the EPOXI ("DIXI") mission encounter with the nucleus of Comet 103P/Hartley 2 (called 103P hereafter), near-aphelion 
observations by Meech et al. (2009) indicated the presence of a periodicity near $16.6 \mathrm{~h}$ in the comet's light-curve, and Lisse et al. (2009) had pointed out that the small size of the nucleus of the comet ( $\sim 0.57 \mathrm{~km}$ effective radius) combined with its relatively large perihelion water production rate $\left(3 \times 10^{28} \mathrm{~mol} / \mathrm{s}\right.$, A'Hearn et al., 1995) implied that the spin state of the nucleus was likely highly excited and might substantially change in a single perihelion passage.

Around the time of encounter (on 2011-11-04 with closest approach at 13:59:47.310, i.e., JD 2455505.0831866), numerous Earth-based time-series observations of structures in the comet's coma (Meech et al., 2011; Knight and Schleicher, 2010, 2011; Samarasinha et al., 2010, 2011; Jehin et al., 2010), radar observations of the nucleus (Harmon et al., 2010, 2011), and high-spectral resolution microwave line observations of HCN (Drahus et al., 2011) indicated that the magnitude of the periodicity was increasing with time and that the spin of 103P's nucleus was probably in an excited state. That this was definitely the case was independently determined in the preliminary approach photometry and imaging results of the DIXI mission (A'Hearn et al., 2011; Belton et al., 2011a). While all of these studies were in broad agreement as to the magnitude and changes in the dominant periodicity and that a second periodicity was present, discussions at a special workshop on the subject held at the Kitt Peak National Observatory on April 5-6, 2011, showed less unanimity on the space direction of $\mathbf{M}$ (the rotational angular momentum per unit mass vector), the magnitude of the roll or oscillation periodicity, or whether the excited motion was in the short (SAM) or long (LAM) axis mode (see the Appendix in Samarasinha and A'Hearn (1991) for a detailed explanation of torque-free excited spin and the SAM and LAM modes).

In this work we present a detailed analysis of DIXI observations that bear on the nucleus spin and show how the spin state changes through the DIXI encounter and that, while the rotation state is definitely excited, it is much less so than anticipated by Lisse et al. (2009). We also determine the average space direction of $\mathbf{M}$ and the motion of the instantaneous spin vector throughout the encounter.

In Section 2 we collect all of the information about the nucleus, derived mainly from DIXI observations, that is needed for the analysis. In Section 3 we present our basic assumptions and an overview of the technique used to determine the spin state and its evolution. In Section 4 we analyze the approach and recession photometry to determine the dominant periodicities in the light-curve and show that there are two groups of periodicities one of which increases with time and the other which decreases (Belton et al., 2011a). In Section 5 we deduce the kinematics of the spin state and evaluate the magnitudes of $\mathbf{M}$ and $E$. In Section 6 we analyze spatially resolved images of the nucleus taken during the time interval Enc(ounter) - $1 \mathrm{~h}$ to Enc using position angle (PA) of the axis of minimum moment of inertia, i.e., the "long" axis, to determine the direction of $\mathbf{M}$ and improved estimates of the intermediate and short principal axes on the sky. In Section 7 we discuss the implications of the changing the estimated direction of the intermediate principal axis of inertia and of the changing spin state (and implied net torques) on conditions in the inner coma including the production of water vapor at the nucleus surface, the orientation of a plume of water vapor, and the time evolution of insolation at active areas.

\section{Relevant properties of the nucleus}

In Table 1 we collect various parameters that describe the orientation of the nucleus at the time of closest approach and are needed to specify the spin state. Most of these quantities have been noted earlier by Thomas et al. (2012), but we repeat them here for convenience. Also needed in this study is a competent model of the shape of the nucleus (Fig. 1). This model, again provided by Thomas et al. (2012), is available on a $2^{\circ}$ longitude-latitude grid with its

Table 1

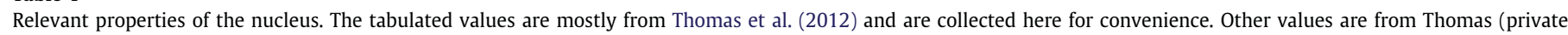
communication). The principal moments of inertia $I_{l}, I_{i}$, and $I_{s}$ are related to the long $(l)$, intermediate $(i)$, and short (s) axes respectively. In this study we use the $I_{l}$ and $I_{i}$ moment

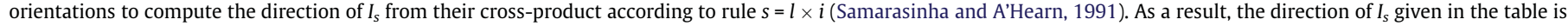

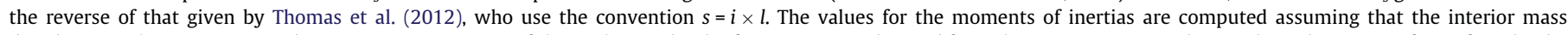
distribution is homogeneous and are given per unit mass of the nucleus. Orbital information was obtained from the JPL HORIZONS web site where the spacecraft is referred to by “@-140”.

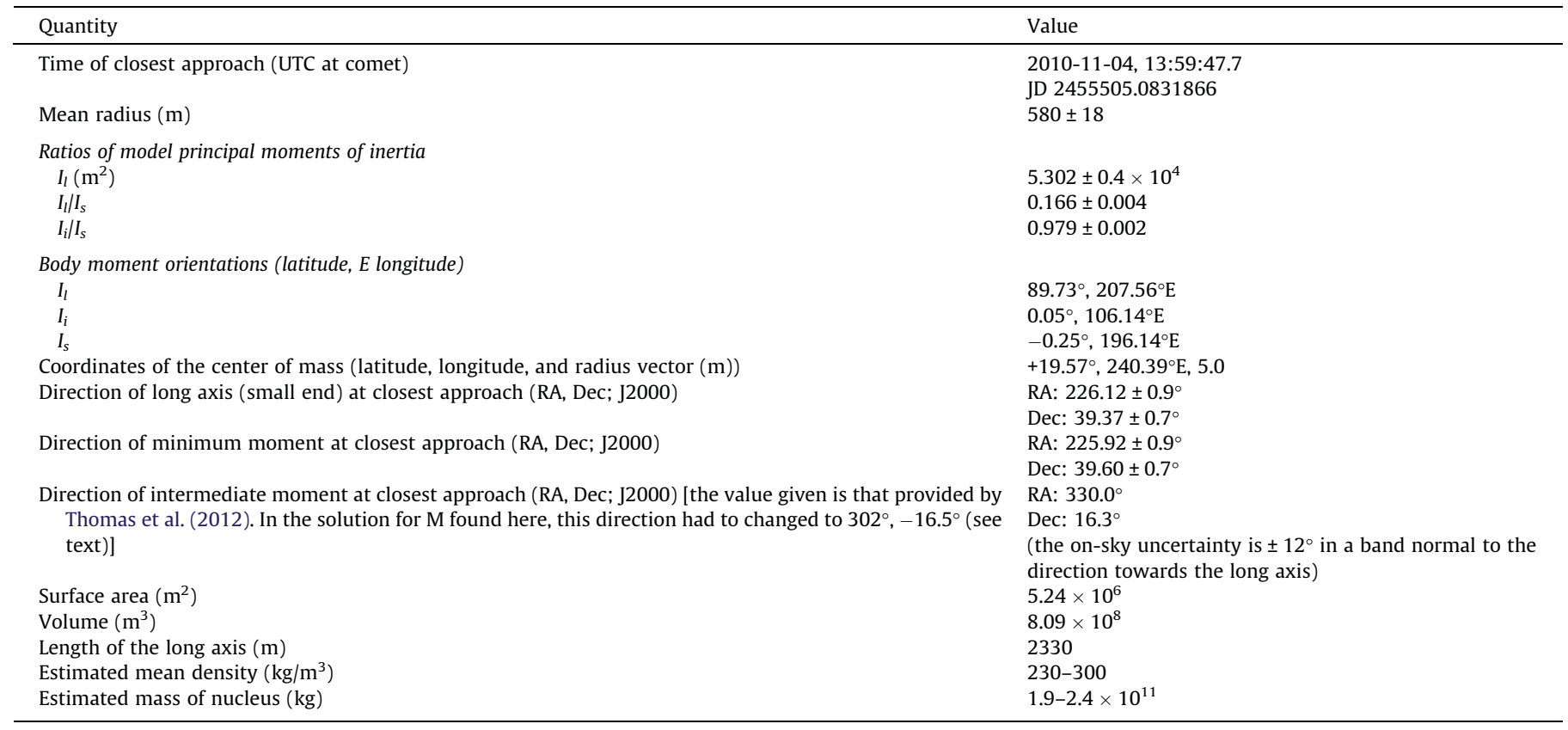




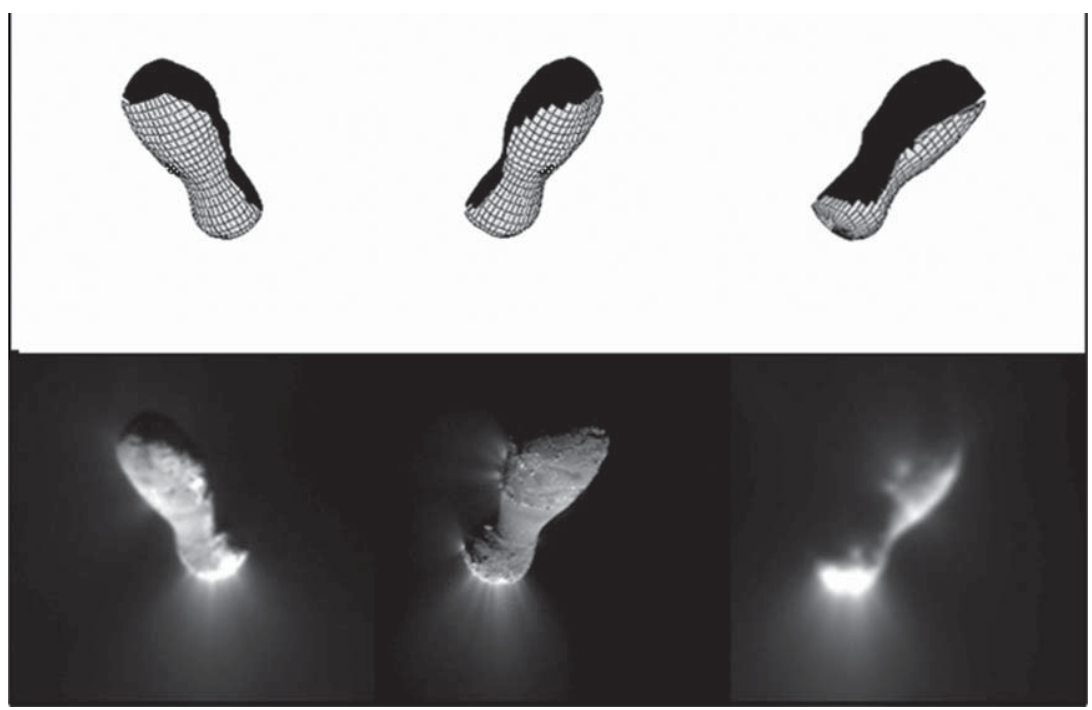

Fig. 1. Comparison of MRI images with model simulations of the nucleus of 103P at E-199.07s, E-0.67s and E+403.88s. The images are mv10110413_5004000, mv10110413_6000002, and mv10110414_5006046. The sub-solar region can be seen as a dark area in the "waist" region of the two left images. The shape of both the nucleus and terminator are simulated reasonably well even though, in this rendition, the scaling (not orientation) of the images and models were approximated by hand. The changing spatial resolution is evident in the images and is a reflection of the different ranges at which the images were taken. From left to right the ranges are: 2548,694 , and 5024 km.

various radius vectors originating near the center of figure. We use this model, as shown in Fig. 1, after its transformation to a cylindrical coordinate system with the origin at the center of mass and coordinate positions referred to the principle axes of inertia. As applied to this problem, we have transformed the model to a coarser grid mainly to reduce computing time and the sizes of the matrices involved (all the calculations in this paper were done in MathCad scripts [Mathsoft Engineering \& Education, Inc.] or in Microsoft Excel spreadsheets). The location of the center of mass assumes a homogeneous interior (see Section 3).

Although our model is relatively coarse, it retains enough information on the shape to display its primary features and, in order to compute the time dependence of solar illumination at any location on the nucleus (Section 7), normals to the local surface in the $5^{\circ}$ grid model were computed at any point by using information from it and the surrounding four grid points.

\section{Overview of method and assumptions used to determine the spin state}

Our basic assumptions are that the mass distribution in the interior of the nucleus is homogeneous and the nucleus rotates as a rigid body. These assumptions were also made and justified for cometary nuclei in an earlier study of the spin of 9P/Tempel 1 (Belton et al., 2011b), and we refer the reader to that work for details. Ultimately, however, the justification rests on how well the model simulates the observed motion, and we will present an example in Section 6. With these assumptions, we can make use of the detailed theoretical description of torque-free asymmetric rigid-body spin developed in the Appendix of Samarasinha and A'Hearn (1991). We will not repeat the lengthy development of their analysis here except to note that we use precisely the same nomenclature, definitions, and system of Euler angles that are used in Samarasinha and A'Hearn's work. When it is necessary to refer to one of their equations, we use the same numbering system as found in Samarasinha and A'Hearn followed by "(SA)." For example, the fundamental equation of motion $(\mathrm{d} \mathbf{M} / \mathrm{d} t)_{\mathrm{ext}}=0$ is Eq. A1(SA).

We begin with the estimation of the periodicities $P_{\bar{\phi}}(t)$ and $P_{\psi}(t)$, which are the time averaged period of circulation of the long axis about $\mathbf{M}$ and the period of roll or oscillation about the long axis at time $t$ respectively. These quantities are evaluated at different times during the encounter by dividing the comet's $81.5 \mathrm{~d}$ lightcurve into sections. The subscripts $\phi, \psi$ (as well as a third, $\theta$ ), refer to the same Euler angles that are defined in Fig. A1 of Samarasinha and A'Hearn (1991). $\dot{\phi}$ and $\dot{\psi}$ refer to the rates at which the long axis circulates about $\mathbf{M}$ and the rate of roll or oscillation about the long axis, respectively. $\theta$ is the angle subtended between the long axis and $\mathbf{M}$, which oscillates with a period of $P_{\psi} / 2$ around a value that we find to slowly decrease throughout the encounter.

The fact that we find that $P_{\bar{\phi}}$ and $P_{\psi}$ vary secularly with time implies that the motion is not torque-free, and this finding requires that we make a further assumption, again to be justified a posteriori. We assume that the actual motion throughout a single spin cycle deviates at most by a small amount from torque free motion. With this assumption we can use the formalism of Samarasinha and A'Hearn (1991) to calculate the spin state at each instant of time with the relevant values of the evolving rotational momentum and energy. In this work the evolution of the spin state is, in effect, assumed to be a progression of torque-free states.

Once $P_{\bar{\phi}}(t)$ and $P_{\psi}(t)$ are known, we can determine which spin mode is appropriate and then estimate the magnitude of $\mathbf{M}(t)$ and the rotational energy, $E(t)$ throughout the encounter. The Euler angles $\psi(t)$ and $\theta(t)$ can also be evaluated at this point.

A first approximation to the space direction of $\mathbf{M}$, which is assumed to be fixed in this study, is then estimated from $\psi(0)$ and $\theta(0)$, and the space directions of the long and intermediate principal axes at closest approach from Thomas et al. (2012), where $t=0$ is the time of closest approach. This estimate, whose uncertainty is dominated by the considerable uncertainty in Thomas et al.'s (2012) estimate of the direction of the intermediate axis, is then improved by applying high-resolution observations of the changing position angles (PA) of the nucleus, measured over as long a time interval as possible, as constraints during this time by minimizing variance between the model and the data.

\section{Determination of $\boldsymbol{P}_{\bar{\phi}}(t)$ and $\boldsymbol{P}_{\psi}(t)$ from encounter light-curves}

Aperture photometry, centered on the nucleus, was performed on 32,049 calibrated images using the IDL "aper.pro" program with a 3-pixel radius aperture. The photometric reductions generally followed the procedures that were used in an earlier study of Co- 

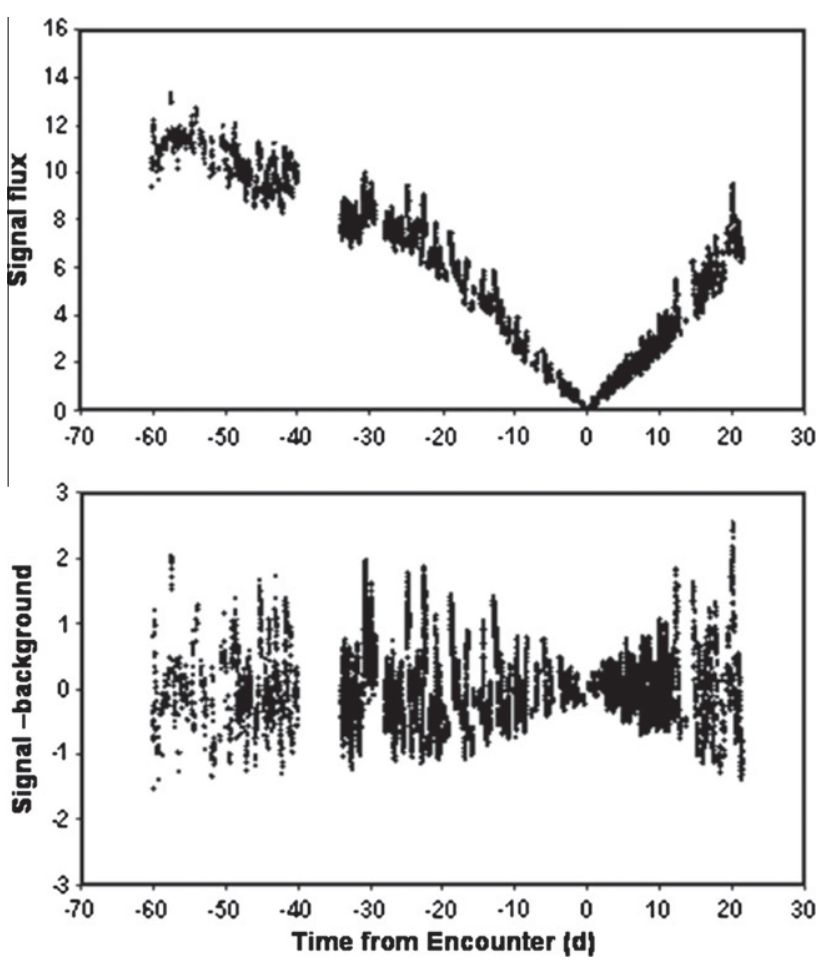

Fig. 2. (Top) 103P light-curve using the MRI camera with the CLEAR1 filter. This data consist of 24,799 points and have been scaled to remove the inverse-square dependence on heliocentric distance and range. (Bottom) Same as the upper panel but with the background removed. The removal process was done in $\sim 10$ day sections (see Table 2).

met 9P/Tempel 1 (Belton et al., 2011b). A more complete description of the overall reduction procedure in provided in Bodewits et al. (2012). Only MRI (Medium Resolution Instrument) images in the CLEAR1 filter, not compromised by cosmic ray hits, saturation, smear, or other evidence of corruption, were used. This left some 24,799 measurements to be used in the analysis (Table S1 in Supplementary Online Data). The data cover $81.6 \mathrm{~d}$ during the period from Enc -60 to Enc +21.6 d. Two versions of the lightcurve, one background subtracted, are shown in Fig. 2. The original data were adjusted to remove the dependence on heliocentric distance and a presumed inverse square dependence on range. Since the signal is a mixture of resolved coma and unresolved nucleus, the latter step is not essential, but its inclusion does not adversely affect the analysis. The data were divided into seven groups on the basis of date rather than number of observations (Table 2). This grouping was done to better spread out the derived periodicities in time, even though the power spectra had very different signalto-noise ratios. Each group was analyzed for latent periodicities

Table 2

Section of data used in the analysis of the light-curve. The median time is defined as the average time of all of the data in the block. The times are measured from the time of closest approach (Table 1).

\begin{tabular}{llllcl}
\hline $\begin{array}{l}\text { Data } \\
\text { section }\end{array}$ & $\begin{array}{l}\text { Start } \\
\text { time }(\mathrm{d})\end{array}$ & $\begin{array}{l}\text { Stop time } \\
\text { (d) }\end{array}$ & $\begin{array}{l}\text { Median } \\
\text { time }(\mathrm{d})\end{array}$ & $\begin{array}{l}\text { Number of } \\
\text { points }\end{array}$ & Sampling \\
\hline 1 & -60.0209 & -48.6907 & -54.5876 & 318 & Sparse \\
2 & -48.6904 & -40.0217 & -44.3603 & 684 & - \\
3 & -33.9018 & -24.1916 & -29.0365 & 3897 & - \\
4 & -23.9018 & -14.2367 & -19.4743 & 4061 & - \\
5 & -13.9018 & -0.2067 & -7.8314 & 4728 & - \\
6 & 0.1878 & 11.9993 & 7.0710 & 10016 & Continuous \\
7 & 12.0003 & 21.5698 & 16.5403 & 1095 & Continuous \\
\hline
\end{tabular}

using the ANOVA technique (Schwarzenberg-Czerny, 1996) as implemented in the commercial Peranso astronomical software package (www.CBABelgium.com). We generally work in the frequency domain (cycles/d) rather than the time domain (periods), because it is much easier to perceive harmonic relationships between individual spectral features in the power spectra in the frequency domain. The ANOVA software, which was used successfully in our study of 9P/Tempel 1, displays the power spectrum and computes the frequency of selected spectral features and their uncertainty. The method of estimating these uncertainties is that described in Schwarzenberg-Czerny (1991). In Fig. 3a and b we show the groups of data and the resulting power spectra.

These spectra show considerable changes in the distribution of power amongst the spectral features (lines) as the encounter progresses. In addition, there are systematic shifts in the peak periods of the lines. This is shown in Table 3 and in Fig. 4. There are two groups of features. One, Group 1, contains three lines, marked as $v_{1}, v_{3}$, and $v_{4}$ in the figure, that generally decrease in frequency as the encounter progresses. The second group, Group 2, contains two lines $\left(v_{2}, v_{5}\right)$ that generally increase in frequency as time progresses. Evidently, these two groups are related to different physical aspects of the rotation. Inspection of the numerical values of the frequency of the lines shows that the lines in each group are harmonically related, i.e., $v_{1} \cong 2 v_{3} \cong 3 v_{4}$ and $v_{2} \cong 3 v_{5}$. This relationship is illustrated as frequency differences that hover around zero in Table 4.

\subsection{Physical identification of the observed frequencies}

Delay-Doppler radar images (Harmon et al., 2011) and a doublepeaked nucleus light-curve (Meech et al., 2009, 2011) definitively relate observations of an increasing period of $16.6-18.2 \mathrm{~h}$, i.e., a decreasing frequency from $\sim 1.45$ to $1.32 \mathrm{c} / \mathrm{d}$, with the circulation (rotation) of the long axis of an elongated nucleus about $\mathbf{M}$ in the interval between April-May, 2009 and during October, 2010. This rate is in good agreement with the magnitude of the change in Group 1 and provides a secure identification of the physical cause of $v_{1}$ : the circulation of the long axis of this elongated body around M. $v_{3}$ and $v_{4}$ are clearly sub-harmonics of $v_{1}$ presumably stimulated by the locations of whatever source(s) of coma material are ultimately responsible the light-variations.

The theory of rigid body rotation indicates that a second group of frequencies is related to either a roll or oscillation about the long axis (Samarasinha et al., 2004). Evidence for a second periodicity has already been seen indirectly in Earth-based observations. Microwave spectroscopy near encounter by Drahus et al. (2011) shows that the pattern of HCN production repeats every three $\sim 18.2 \mathrm{~h}$ cycles, and Knight and Schleicher (2011), studying coma structures, found that "the best rotational matches were found for images multiples of three cycles apart." More recently, Waniak et al. (2012) have found that their images of the central coma also have a three-cycle repetition. In addition, a similar 3-cycle pattern can be seen in the post-encounter DIXI data, e.g., in the CLEAR1 filter light-curve (Fig. 3b) for the 12 days immediately following closest approach. Evidently, the rotation of the nucleus brings the sources on the nucleus to essentially the same configuration with respect to the Sun every three-cycles, i.e., every $54-55 \mathrm{~h}$ or with a frequency near $0.44 \mathrm{c} / \mathrm{d}$. We can be reasonably assured of this result if we identify $v_{2}$, but not $v_{5}$, as the frequency of roll or oscillation about the long axis since, as seen in Table 5, $\left\{v_{1}\right\} /\left\{v_{2}\right\}$ averages 1.5 throughout the encounter. $v_{5}$ is therefore a sub-harmonic of $v_{2}$, i.e., $v_{2}=3 v_{5}$ (Table 4 ).

In summary, we identify $v_{1}$ with the circulation of the long axis around $\mathbf{M}$ and $v_{2}$ with the roll or oscillation of the nucleus around its long axis. In order to make maximum use of the information in the power spectra and the precision of the harmonic relationships 

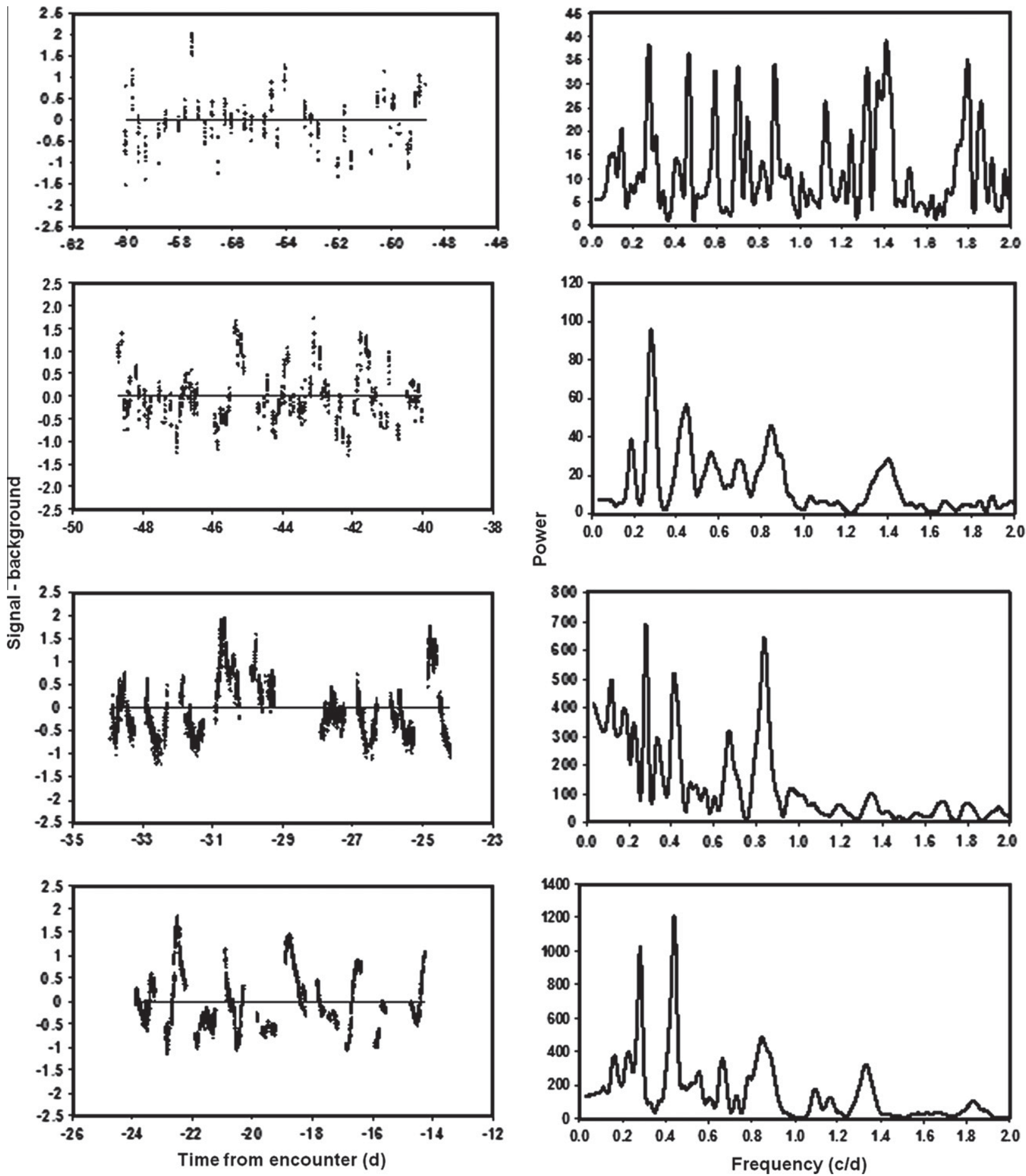

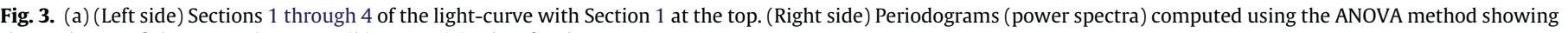
the evolution of the spectral content. (b) As in 3(a) - but for data Sections 5-7.

seen in Table 4, we combine the information of all the lines within each group to form a weighted average $\left\{v_{1}\right\}(t)$ and $\left\{v_{2}\right\}(t)$ at each time step. Thus, $P_{\bar{\phi}}=\left\{v_{1}\right\}^{-1}$ and $P_{\psi}=\left\{v_{2}\right\}^{-1}$. The resulting values are collected in Table 5 , and the time-evolutions of $\left\{v_{1}\right\}(t),\left\{v_{2}\right\}(t)$, $P_{\bar{\phi}}(t)$ and $P_{\psi}(t)$ around the time of encounter are shown in Fig. 5. We emphasize that the relationship between $\left\{v_{1}\right\}$ and $\left\{v_{2}\right\}$ is approximate and varies slowly throughout the encounter. It is not, apparently, a constant of the motion or indicative of a reso- nance. The period of circulation of the long axis is seen to steadily increase through perihelion passage in an approximately linear fashion at a rate of $\sim 1.3 \pm 0.2 \mathrm{~min} / \mathrm{d}$ in agreement with what was found in the Earth-based observations of Drahus et al. (2011) and Knight and Schleicher (2011). The evolution of the roll around the long axis is more complex, with the angular rate apparently slowing down at first until about $30 \mathrm{~d}$ before perihelion and then speeding up through perihelion passage. However, the reality of 

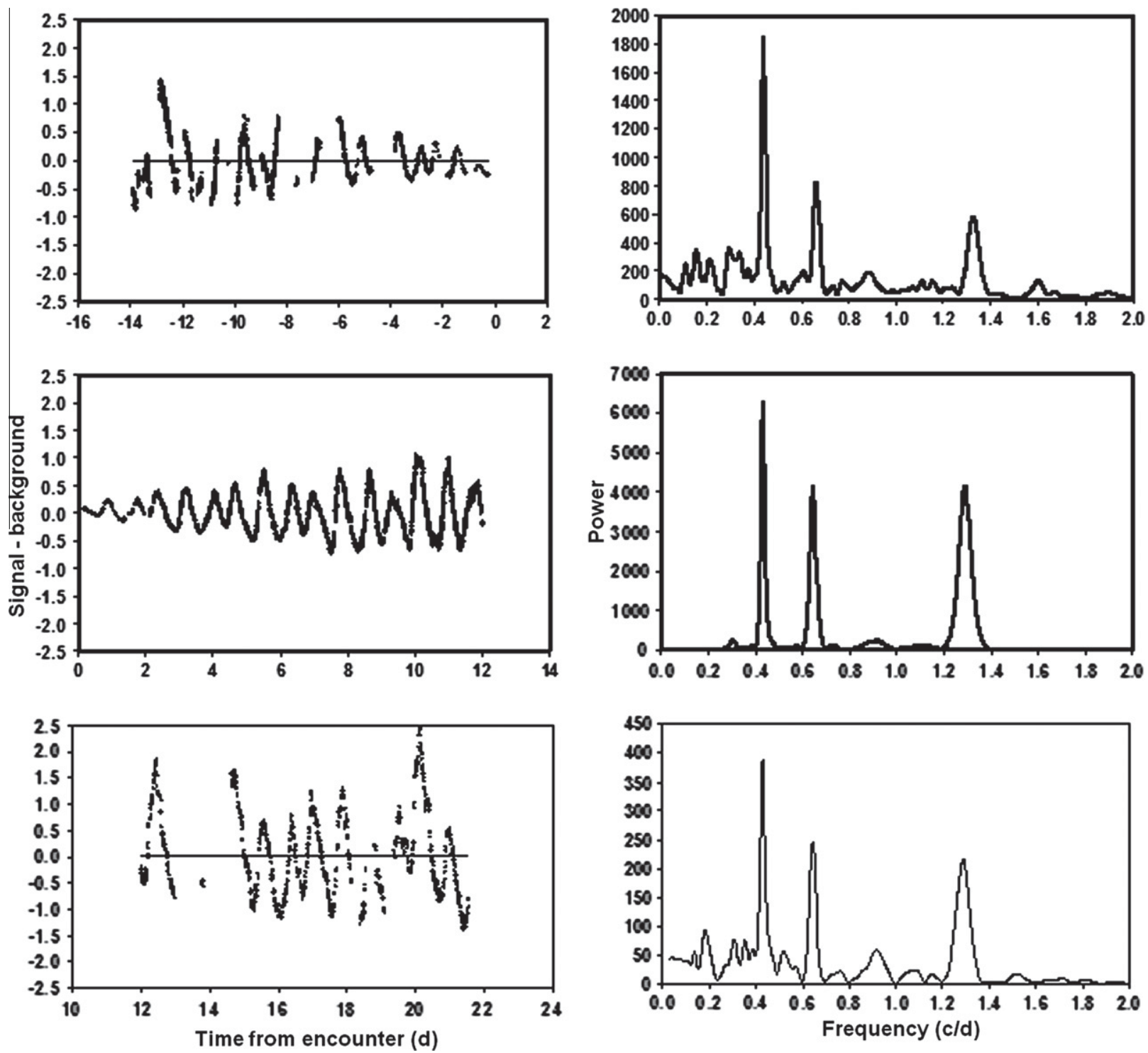

Fig. 3 (continued)

this early slow down is questionable since the evidence rests on the value of a single point. The rate of change of roll period is approximately $-4.4 \mathrm{~min} / \mathrm{d}$ at perihelion. There is a hint in the data that the rate of change of roll is beginning to stabilize some $20 \mathrm{~d}$ after perihelion, but this conclusion is obviously very uncertain.

\section{Kinematics of $103 \mathrm{P} /$ Hartley 2 's spin state}

We look at the kinematics of 103P's spin in two parts. In the first we determine the mode in which the nucleus is spinning and compute $\mathbf{M}$ (the magnitude of $\mathbf{M}$ ), $E$ and other parameters of the spin state based on the above values of $P_{\bar{\phi}}$ and $P_{\psi}$. In the second we determine our best estimation of the space orientation of the spin state using PAs of the long axis of the nucleus measured in preencounter observations.

\subsection{The mode of spin}

The question of what spin mode the nucleus finds itself in during the encounter depends not only on $P_{\bar{\phi}}$ and $P_{\psi}$ but also on the principal moments of inertia $I_{l}, I_{i}, I_{s}$ (Table 1). Samarasinha and A'Hearn (1991, Appendix) have developed two inequalities, where the value of the ratio $P_{\psi} / P_{\bar{\phi}}$ relative to functions of the principal moments of inertias, determines whether the spin mode is a LAM or SAM.

For a LAM we must have:

$\frac{P_{\psi}}{P_{\bar{\phi}}} \geqslant\left[\sqrt{\frac{I_{i} I_{s}}{\left(I_{i}-I_{l}\right)\left(I_{s}-I_{l}\right)}}-1\right]$

and, for a SAM:

$\frac{P_{\psi}}{P_{\bar{\phi}}} \geqslant\left[\sqrt{\frac{I_{i} I_{l}}{\left(I_{s}-I_{i}\right)\left(I_{s}-I_{l}\right)}}-1\right]$

$(\mathrm{A} 78(\mathrm{SA}))$

In Fig. 6 we apply these inequalities to the situation for 103P. Throughout the encounter the spin state is seen to lie firmly in the LAM region, with the decrease in this ratio with time indicating an increase in the level of excitation as the encounter progresses. Also shown in the figure are the effects of the level of uncertainty 
Table 3

Evolution of line periodicities (h) through the encounter.

\begin{tabular}{|c|c|c|c|c|c|}
\hline \multirow[t]{2}{*}{ Time from encounter $(d)$} & \multicolumn{5}{|c|}{ Periodicies $(\mathrm{h})$} \\
\hline & $v_{1}$ & $v_{2}$ & $v_{3}$ & $v_{4}$ & $v_{5}$ \\
\hline-54.5877 & $17.05 \pm 0.23$ & $27.42 \pm 0.37$ & $34.28 \pm 0.57$ & $51.57 \pm 0.86$ & $87.09 \pm 2.59$ \\
\hline-44.3603 & $17.10 \pm 0.78$ & $28.28 \pm 0.80$ & $34.28 \pm 1.51$ & $53.69 \pm 5.76$ & $86.01 \pm 6.07$ \\
\hline-29.0365 & $17.83 \pm 0.59$ & $28.62 \pm 0.39$ & $35.61 \pm 0.95$ & $58.16 \pm 2.24$ & $86.14 \pm 1.77$ \\
\hline-19.4743 & $18.02 \pm 0.06$ & $28.28 \pm 0.13$ & $36.11 \pm 0.16$ & $54.46 \pm 0.14$ & $84.66 \pm 0.32$ \\
\hline-7.8315 & $18.12 \pm 0.05$ & $27.13 \pm 0.28$ & $36.17 \pm 0.08$ & $54.36 \pm 0.07$ & $76.29 \pm 6.16$ \\
\hline 7.0711 & $18.63 \pm 0.02$ & $26.32 \pm 0.26$ & $37.26 \pm 0.03$ & $55.76 \pm 0.04$ & $79.00 \pm 0.68$ \\
\hline 16.5404 & $18.61 \pm 0.09$ & $26.13 \pm 0.23$ & $37.28 \pm 0.24$ & $55.92 \pm 0.30$ & $78.19 \pm 2.09$ \\
\hline
\end{tabular}
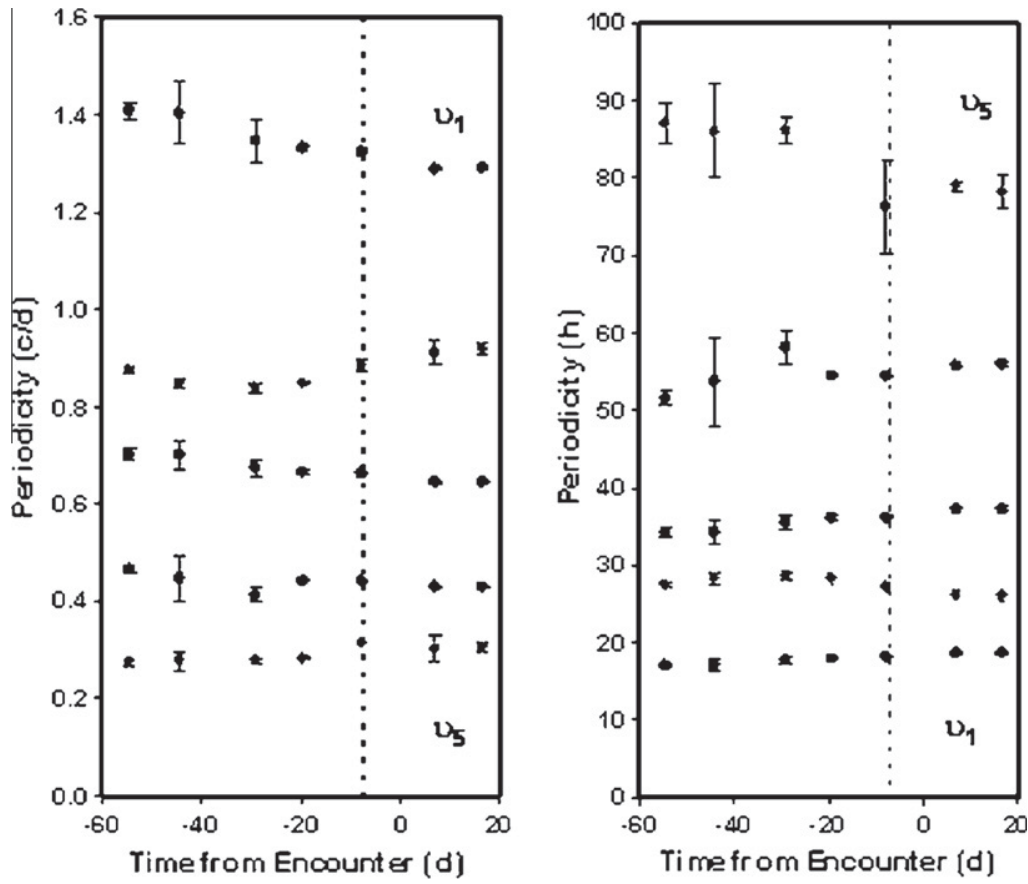

Fig. 4. (Left) Evolution of the frequencies of the five most prominent lines in the power spectra of the seven blocks of data. The lines are labeled in order: $v_{1}$ through $v_{5}$ However, for clarity of presentation, we have labeled only the first and last lines in the Figure. (Right) Evolution of the periods of the five lines. In both parts of the figure. two groups of lines can be identified: $\left(v_{1}, v_{3}, v_{4}\right)$ and $\left(v_{2}, v_{5}\right)$. In the first group the frequencies of the lines decrease with time through perihelion passage. In the second the frequencies increase with time. Perihelion passage (JD 2455497.756967) is marked as a vertical dotted line.

Table 4

Frequency (c/d) differences between base line frequency and their sub-harmonics in the two groups of lines. In most cases the magnitude of the differences are substantially smaller than the uncertainty in the base frequency.

\begin{tabular}{|c|c|c|c|c|c|c|}
\hline \multicolumn{4}{|l|}{ Group 1} & \multicolumn{3}{|l|}{ Group 2} \\
\hline$v_{1}$ & Uncertainty & $2 v_{3}-v_{1}$ & $3 v_{4}-v_{1}$ & $v_{2}$ & Uncertainty & $3 v_{5}-v_{2}$ \\
\hline 1.28932 & 0.00592 & -0.0016 & -0.00178 & 0.91844 & 0.00824 & 0.00235 \\
\hline 1.28846 & 0.00114 & $-1 \mathrm{E}-04$ & 0.00283 & 0.91179 & 0.00902 & -0.00042 \\
\hline 1.32436 & 0.00344 & 0.00264 & $8 \mathrm{E}-05$ & 0.88457 & 0.00924 & 0.05923 \\
\hline 1.33204 & 0.00464 & -0.00272 & -0.01012 & 0.84868 & 0.00396 & 0.00182 \\
\hline 1.34592 & 0.0448 & 0.00196 & -0.10794 & 0.83864 & 0.01142 & -0.00281 \\
\hline 1.40384 & 0.0642 & -0.00356 & -0.06287 & 0.84852 & 0.02396 & -0.0114 \\
\hline 1.40737 & 0.01917 & -0.00697 & -0.01123 & 0.87541 & 0.01194 & -0.04867 \\
\hline
\end{tabular}

in the moments of inertia. Only if there were significant large-scale axial inhomogeneities in the internal mass distribution in the nucleus would it be possible for the nucleus to be in a SAM state, i.e., even though the nucleus is close to axial symmetry, the moments $I_{i}$ and $I_{s}$ would have to have radically different values. Further investigation on this point is beyond the scope of this work, and we conclude that the nucleus of 103P spins in the long-axis mode with the level of excitation increasing through the time of closest approach.
5.2. Determination of rotational angular momentum and energy of the spin

As shown in the Appendix to Samarasinha and A'Hearn (1991) we can set up definitions for the observed $P_{\psi}$ and $P_{\bar{\phi}}$ in terms of $\mathbf{M}$ and $E$, i.e.

$\left\langle P_{\bar{\phi}}\right\rangle=2 \pi /\left[\left(2 / P_{\psi}\right) \int_{0}^{P_{\psi} / 2} \dot{\phi} d t\right] \quad$ (SA not numbered) 
Table 5

Weighted estimates of the period (frequency) of circulation of the long axis about $\mathbf{M}$ and the roll period (frequency) throughout the encounter.

\begin{tabular}{|c|c|c|c|c|c|c|c|c|c|}
\hline Time from encounter (d) & $\left\{v_{1}\right\}(\mathrm{c} / \mathrm{d})$ & $\Delta v_{1}( \pm)$ & $P_{\bar{\phi}}(\mathrm{h})$ & $\Delta P_{\bar{\phi}}( \pm)$ & $\left\{v_{2}\right\}(\mathrm{c} / \mathrm{d})$ & $\Delta v_{2}( \pm)$ & $P_{\psi}(\mathrm{h})$ & $\Delta P_{\psi}( \pm)$ & $\left\{v_{1}\right\} /\left\{v_{2}\right\}$ \\
\hline-54.58 & 1.4022 & 0.0125 & 17.12 & 0.15 & 0.8661 & 0.0107 & 27.71 & 0.34 & 1.618 \\
\hline-44.36 & 1.3967 & 0.0425 & 17.18 & 0.52 & 0.8469 & 0.0222 & 28.34 & 0.74 & 1.649 \\
\hline-29.03 & 1.3193 & 0.0242 & 18.19 & 0.33 & 0.8378 & 0.0095 & 28.65 & 0.33 & 1.574 \\
\hline-19.47 & 1.3260 & 0.0025 & 18.10 & 0.03 & 0.8498 & 0.0025 & 28.24 & 0.08 & 1.560 \\
\hline-7.83 & 1.3249 & 0.0013 & 18.11 & 0.02 & 0.8854 & 0.0092 & 27.11 & 0.28 & 1.496 \\
\hline 7.07 & 1.2897 & 0.0006 & 18.61 & 0.01 & 0.9116 & 0.0059 & 26.33 & 0.17 & 1.414 \\
\hline 16.54 & 1.2884 & 0.0039 & 18.63 & 0.06 & 0.9187 & 0.0078 & 26.12 & 0.22 & 1.402 \\
\hline
\end{tabular}

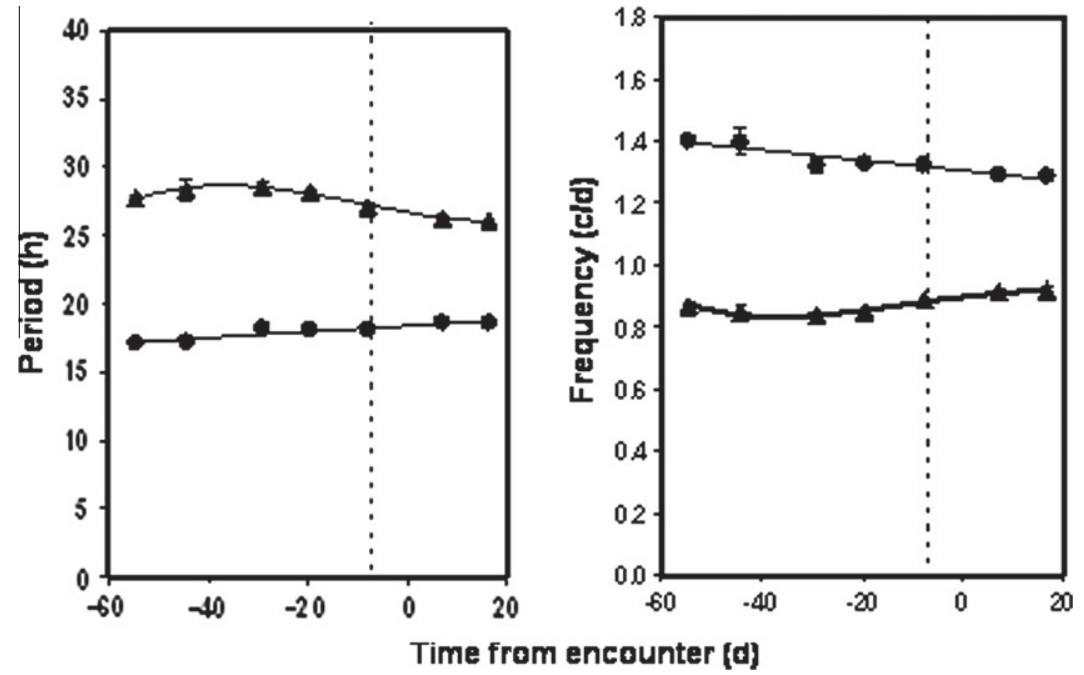

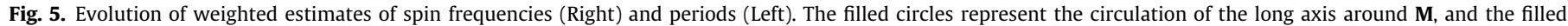

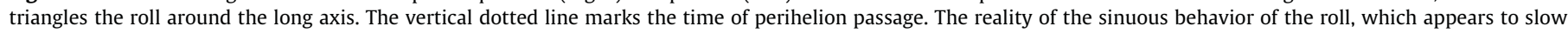
down at first and then speed up through perihelion, is uncertain, as it depends on a single point. Only the trend through perihelion is secure.

where

$\dot{\phi}=M\left[\frac{\left.\left(I_{i}-I_{l}\right)+\left(I_{s}-I_{i}\right) * s n^{2} \tau\right)}{I_{s}\left(I_{i}-I_{l}\right)+I_{l}\left(I_{S}-I_{i}\right) s n^{2} \tau}\right] \quad(\mathrm{A} 38(\mathrm{SA}))$

and

$P_{\psi}=4 \sqrt{\frac{I_{l} I_{i} I_{s}}{2 E\left(I_{i}-I_{l}\right)\left(I_{S}-\frac{M^{2}}{2 E}\right)}} \int_{0}^{\pi / 2} \frac{d u}{\sqrt{1-k^{2} \sin ^{2} u}} \quad(\mathrm{~A} 45(\mathrm{SA}))$

where

$k^{2}=\frac{\left(I_{s}-I_{i}\right)\left(\frac{M^{2}}{2 E}-I_{l}\right)}{\left(I_{i}-I_{l}\right)\left(I_{s}-\frac{M^{2}}{2 E}\right)} \quad(\mathrm{A} 32(\mathrm{SA}))$

and

$\tau=t \sqrt{\frac{2 E\left(I_{i}-I_{l}\right)\left(I_{s}-\frac{M^{2}}{2 E}\right)}{I_{i} I_{i} I_{S}}} \quad(\mathrm{~A} 31(\mathrm{SA}))$

In Eq. (3) the integration is taken over $P_{\psi} / 2$, because it is one of the peculiarities of rigid body motion that $P_{\bar{\phi}}$ is periodic over this interval. Eqs. (3) and (5) are non-linear but can be solved numerically if we have good first approximations for $\mathbf{M}$. and $E$. We get a first approximation to the magnitude of $\mathbf{M}$, i.e., $M$, from

$\left(P_{\bar{\phi}}\right)_{\min } \rightarrow \frac{2 \pi I_{i}}{M} \quad(\mathrm{~A} 49(\mathrm{SA}))$

where the arrow indicates the limiting value, and

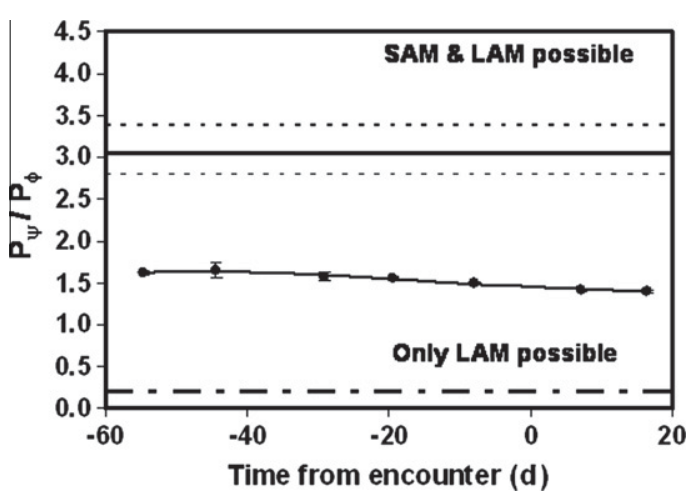

Fig. 6. Determination of the mode of spin. The ratio $P_{\psi} P_{\bar{\phi}}$ falls entirely in the area where LAM states are allowed (above the dash-dot line defined by Eq. (1)). The (unbroken) horizontal line, shown with its uncertainty level, is defined by Eq. (2). Only above this line are SAM states allowed in this system.

$\left(P_{\bar{\phi}}\right)_{\max }=\frac{2 \pi I_{l} \sqrt{I_{i} I_{S}}}{M\left[\sqrt{I_{i} I_{S}}-\sqrt{\left.\left(I_{i}-I_{l}\right)\left(I_{S}-I_{l}\right)\right]}\right.} \quad(\mathrm{A} 50(\mathrm{SA}))$

by putting the observed value of $P_{\bar{\phi}}$ equal to the functional form of $\left(\left(P_{\bar{\phi}}\right)_{\min }+\left(P_{\bar{\phi}}\right)_{\max }\right) / 2$ and solving for $\mathrm{M}$. Using this value and the observed value for $P_{\psi}$ in Eq. (5), we compute a first approximation to $E$. With these estimates as starting values we can then solve Eqs. (3) and (5) iteratively for precise values of $E$ and $M$. In this study we use the MathCad "solve block" Find function to perform this iteration. The results of this process are collected in Table 6, which 
Table 6

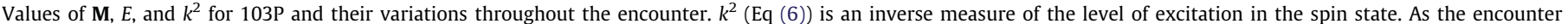

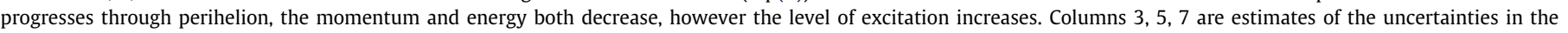
various quantities.

\begin{tabular}{|c|c|c|c|c|c|c|}
\hline Time from encounter (d) & $\mathbf{M}\left(\mathrm{m}^{2} / \mathrm{s}\right)$ & $\Delta \mathbf{M}( \pm)$ & $E\left(\mathrm{~m}^{2} / \mathrm{s}^{2}\right)$ & $\Delta E( \pm)$ & $k^{2}$ & $\Delta\left(k^{2}\right)( \pm)$ \\
\hline-44.36 & $3.208 \mathrm{E}+01$ & $6.90 \mathrm{E}-01$ & $1.750 \mathrm{E}-03$ & $7.03 \mathrm{E}-05$ & $2.454 \mathrm{E}-01$ & 0.201 \\
\hline-29.04 & $3.030 \mathrm{E}+01$ & $3.92 \mathrm{E}-01$ & $1.572 \mathrm{E}-03$ & $3.77 \mathrm{E}-05$ & $2.262 \mathrm{E}-01$ & 0.078 \\
\hline-19.47 & $3.046 \mathrm{E}+01$ & $3.98 \mathrm{E}-02$ & $1.590 \mathrm{E}-03$ & $3.87 \mathrm{E}-06$ & $2.225 \mathrm{E}-01$ & 0.006 \\
\hline-7.83 & $3.043 E+01$ & $2.13 \mathrm{E}-02$ & $1.598 \mathrm{E}-03$ & $2.82 \mathrm{E}-06$ & $2.063 \mathrm{E}-01$ & 0.003 \\
\hline 0 & $2.996 \mathrm{E}+01$ & $1.48 \mathrm{E}-01$ & $1.557 \mathrm{E}-03$ & $1.40 \mathrm{E}-05$ & $1.954 \mathrm{E}-01$ & 0.020 \\
\hline 7.071 & $2.963 \mathrm{E}+01$ & $9.55 \mathrm{E}-03$ & $1.529 \mathrm{E}-03$ & $1.57 \mathrm{E}-06$ & $1.864 \mathrm{E}-01$ & 0.001 \\
\hline 16.54 & $2.959 \mathrm{E}+01$ & $6.39 \mathrm{E}-02$ & $1.529 \mathrm{E}-03$ & $6.23 \mathrm{E}-06$ & $1.833 \mathrm{E}-01$ & 0.007 \\
\hline
\end{tabular}

again shows that the excitation of the spin state is actually increasing through perihelion even though the total rotational momentum and energy are steadily decreasing. The trends in $M, E$, and $k^{2}$ (Eq. (6), an inverse measure of excitation) are shown in Figs. 7 and 8 .

\subsection{Determination of other spin parameters at closest approach}

Once $\mathrm{M}$ and $E$ are determined other parameters that are a function of time can be calculated according to Eqs. A31(SA)-A38(SA). These parameters include two of the Euler angles ( $\psi$ and $\theta$ ), the components of the total spin vector, and the (variable) rate at which the long axis circulates around $\mathbf{M}$. We leave the determination of most of these quantities to the following section where, we estimate the orientation of $\mathbf{M}$ and the nucleus in space. However, there are aspects of the motion that depend on the degree of excitation and exhibit secular change throughout the encounter, which we evaluate here. These quantities include the maximum and minimum values of $\theta$, the Euler angle between the long axis and $\mathbf{M}$, and the maximum and minimum values of the roll rate, $\dot{\psi}$, and the circulation rate, $\dot{\phi}$. The secular evolution of these quantities throughout the encounter is shown in Table 7 . The mean value of $\theta$ steadily decreases from $82.8^{\circ}$ to $81.7^{\circ}$ (another indication of increasing excitation), while the full amplitude of the associated "nodding" motion, which has a period of $P_{\psi} / 2$, decreases from $\sim 1^{\circ}$ to $0.8^{\circ}$. The rate of roll and circulation are shown to vary within each spin cycle by $14-10 \%$ and by about $2 \%$ respectively.

\section{Determination of the orientation of the spin state in space}

As we noted in the introduction, there is little consensus in the various studies of coma morphology on the space direction of $\mathbf{M}$ for 103P. This scatter is shown in Table 8. In this work we have the benefit of the radar observations of Harmon et al. (2011), which show definitively that the circulation of the long axis is in the prograde sense, and the determination of the space directions of the minimum and intermediate axes at the time of closest encounter (Thomas et al., 2012). We note at this point that the sense of roll around the long axis (anti-clockwise looking at the small-end of the nucleus along the long axis) is fixed once the sense of the motion about $\mathbf{M}$ is determined (Ames and Murnaghan, 1929). Together with the Euler angles, specifically $\theta(0)$ and $\psi(0)$, it is a matter of simple spherical trigonometry to estimate the space direction of $\mathbf{M}$ at that time.

The Euler angles at time $t$ are calculated from:

$\psi(t)=\operatorname{atan} 2\left(\sqrt{\frac{I_{i}}{\left(I_{i}-I_{l}\right)}} \operatorname{sn} \tau(t, k), \sqrt{\frac{I_{s}}{\left(I_{s}-I_{l}\right)}} \operatorname{cn} \tau(t, k)\right)$

where the order of the parameters in the atan2 function is as defined in Fortran,
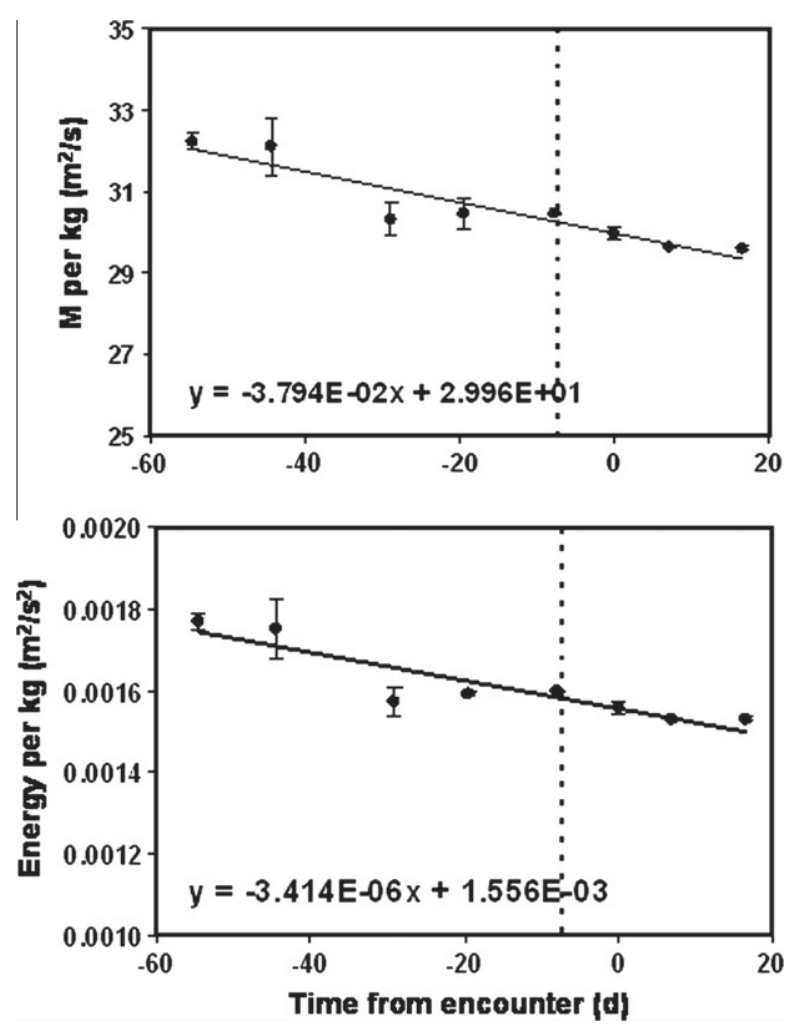

Fig. 7. (Top) The evolution of $\mathbf{M}$ through the encounter. The rotational momentum is seen to decrease steadily through perihelion passage. (Bottom) The rotational energy also decreases through perihelion passage, which is marked with a vertical dotted line.

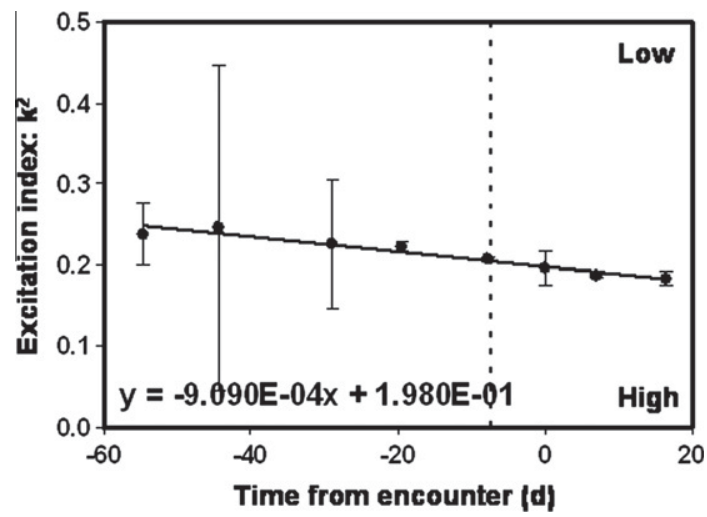

Fig. 8. The index $k^{2}$ (Eq. (6), an inverse measure of the degree of excitation) shows that the spin state moves to a higher excitation level as the encounter passes through perihelion, which is marked with a vertical dotted line. 
Table 7

Secular variation of $\theta$, and $\phi, \psi$ motions throughout the encounter.

\begin{tabular}{|c|c|c|c|c|c|c|}
\hline Time from encounter (d) & $\theta_{\min }\left(^{\circ}\right)$ & $\theta_{\max }\left({ }^{\circ}\right)$ & $\dot{\psi}_{\min }(\mathrm{c} / \mathrm{d})$ & $\dot{\psi}_{\max }(\mathrm{c} / \mathrm{d})$ & $\dot{\phi}_{\min }(\mathrm{c} / \mathrm{d})$ & $\dot{\phi}_{\max }(\mathrm{c} / \mathrm{d})$ \\
\hline-44.36 & 82.47 & 83.46 & 0.7866 & 0.9095 & 1.3812 & 1.4109 \\
\hline-29.04 & 82.16 & 83.11 & 0.7833 & 0.8943 & 1.3047 & 1.3327 \\
\hline-19.47 & 82.10 & 83.04 & 0.7955 & 0.9060 & 1.3114 & 1.3396 \\
\hline-7.83 & 81.80 & 82.70 & 0.8333 & 0.9394 & 1.3104 & 1.3385 \\
\hline 0 & 81.57 & 82.45 & 0.8484 & 0.9498 & 1.2899 & 1.3176 \\
\hline 7.07 & 81.37 & 82.23 & 0.8633 & 0.9612 & 1.2756 & 1.3030 \\
\hline 16.54 & 81.30 & 82.15 & 0.8710 & 0.9679 & 1.2743 & 1.3016 \\
\hline
\end{tabular}

$\theta(t)=\cos ^{-1}\left[d n \tau(t, k) \sqrt{\frac{I_{l}\left(I_{s}-M^{2} / 2 E\right)}{\left(M^{2} / 2 E\right)\left(I_{s}-I_{l}\right)}}\right] \quad(\mathrm{A} 37(\mathrm{SA}))$

and

$\phi(t)=\int_{0}^{t} \dot{\phi} d t$

$\dot{\phi}(t)$ is given in Eq. (4), and $s n \tau, c n \tau$ and $d n \tau$ are Jacobian elliptic functions. $\phi(0)$ is determined once the direction of $\mathbf{M}$ has been calculated from $\psi(0), \theta(0)$ and the directions of the minimum and intermediate axes of moment of inertia. In order to calculate $\phi(0)$ we also choose to orient the $X$-axis of the angular momentum coordinate system (Fig. A1 in Samarasinha and A'Hearn (1991)) into the meridian plane of the Equatorial coordinate system that contains $\mathbf{M}$. At closest approach we find $\phi(0)=241.6^{\circ}, \psi(0)=0^{\circ}$ and $\theta(0)=81.60^{\circ}$, and given the direction of the inertia axes in Table 1 , we find our first approximation to the space direction of $\mathbf{M}$ as (RA, Dec; J2000) $=83.18^{\circ},+53.39^{\circ}$.

The uncertainty in this approximation to the direction of $\mathbf{M}$ is substantial, since it originates in the combined uncertainties in our estimates of the moments of inertia, $\mathbf{M}, E$, and the directions of the long and intermediate axes. The uncertainty in the latter is particularly severe, since according to Thomas et al. (2012) it amounts to $\pm 12^{\circ}$ in a band normal to the direction of $I_{l}$ (the long and intermediate axes are, by definition, orthogonal), so the most likely values of the RA and Dec of $\mathbf{M}$ lie on a highly elongated area across the sky centered on the first approximation as shown in Fig. 9. Deconvolved DIXI high-resolution images (Lindler et al., 2012) provide a powerful constraint here by providing, through measurements the PAs of the long axis over an arc of $\sim 20^{\circ}$ on the sky during $\sim 1 \mathrm{~h}$ of the time before closest approach, a measure of the orientation of the track of the rotating long axis on the sky.

In Fig. 10 we show a sample of deconvolved HRI (High-Resolution Imager) images taken in the period Enc $-1 \mathrm{~h}$ to Enc $+1 \mathrm{~h}$. In our determination of the PA of the long axis we found that only images in the interval Enc $-1 \mathrm{~h}$ to Enc were found suitable owing to the peculiar illumination of the terminator region in the postencounter images (Fig. 10). In Table 9 we list the images that were used and our estimated PAs. These estimates are the mean of three independent sets of measurements by two individuals. Based on the dispersion in these measurements, we assign an uncertainty of $\pm 1.7^{\circ}$ to the PAs. The results are plotted in Fig. 11, which show how the projected orientation of the nucleus changes on approach, but still well in advance of the time of closest approach when the changes are dominated by the spacecraft orbit. Also shown in Fig. 11 is a curve that shows the predicted change in PA based on the first approximation of the direction of $\mathbf{M}\left(X=0^{\circ}\right)$. A clear divergence exists between the predictions of this first approximation and the observed PAs. The origin of this discrepancy is, as shown in the figure, the large uncertainty associated with the direction of the intermediate principal axis of inertia. In the figure we test the result of moving the direction of the intermediate axis along its arc of uncertainty by $X^{\circ}$ (Fig. 9). An excellent account of the observed PAs is found for $X=43^{\circ}$ (Fig. 11), where $\mathbf{M}$ is directed towards (RA, Dec; J2000) $=8 \pm 4^{\circ},+54 \pm 1^{\circ}$. This our best estimate of the direction of $\mathbf{M}$ (equivalent to an obliquity of $48 \pm 1^{\circ}$ ), which determines the orientation of the state. Fig. 12 shows change of variance with $X$ in this determination.

The space direction of the instantaneous spin vector at this time is $(R A, D e c ; J 2000)=300 \pm 3^{\circ},+67 \pm 1.3^{\circ}$. The "adjusted" direction of the intermediate principal axis of inertia is (RA, Dec; $\mathrm{J} 2000)=301.8^{\circ},-16.5^{\circ}$, some $33^{\circ}$ away (on the sky) from the Thomas et al. (2012) estimate, and the corresponding adjustments to the initial Euler angles gives $\phi(0)=298.76^{\circ}, \psi(0)=0^{\circ}$, and $\theta(0)=81.60^{\circ}$. The direction of the short principal axis of inertia must therefore be moved to $14.1^{\circ},+45.8^{\circ}$ for consistency. The instantaneous spin vector is inclined at an angle of $33.2 \pm 1.3^{\circ}$ to $\mathbf{M}$, and the instantaneous spin period is $14.1 \pm 0.3 \mathrm{~h}$ at closest approach. Fig. 13 shows the alignment of the model and observation for the last resolved HRI image after encounter for our best estimate of the spin state.

\section{Discussion and conclusions}

\subsection{Summary of the derived spin state and its evolution}

At the time of closest approach the nucleus is found to spin in the long-axis mode (LAM) with the long axis circulating around $\mathbf{M}$ with a period near $18.40 \pm 0.13 \mathrm{~h}$, tilted to $\mathbf{M}$ near an angle of $81.2 \pm 0.6^{\circ}$, while simultaneously rolling around the long axis with a period of $26.72 \pm 0.06 \mathrm{~h}$. The motion is in the prograde sense. As an alternative description, the instantaneous spin vector moves through the body of the nucleus circulating around $\mathbf{M}$ every $18.40 \pm 0.13 \mathrm{~h}$, while tilted near an angle of $33.2 \pm 1.3^{\circ}$ and rotating with an instantaneous period near $14.1 \pm 0.3 \mathrm{~h}$. These periodicities and tilts are not constant during the motion and vary by a few

Table 8

Estimates of the direction of $\mathbf{M}$.

\begin{tabular}{|c|c|c|c|c|}
\hline Reference & Observation type & $\mathrm{RA}\left({ }^{\circ}\right)$ & $\operatorname{Dec}\left({ }^{\circ}\right)$ & Notes \\
\hline Knight and Schleicher (2011) & Coma features & 257 & +67 & - \\
\hline Samarasinha et al. (2010) & Coma features & $345 \pm 20$ & $-15 \pm 20$ & - \\
\hline Waniak et al. (2012) & Coma features & 122 & +16 & - \\
\hline Harmon et al. (2011) & Radar & 17 & 47 & Consistency \\
\hline P.A Taylor (Private Communication) & Radar & $347 \pm 20$ & $+27 \pm 20$ & Preliminary \\
\hline Belton et al. (this work) & Nucleus imaging & $8 \pm 4$ & $54 \pm 1$ & - \\
\hline
\end{tabular}




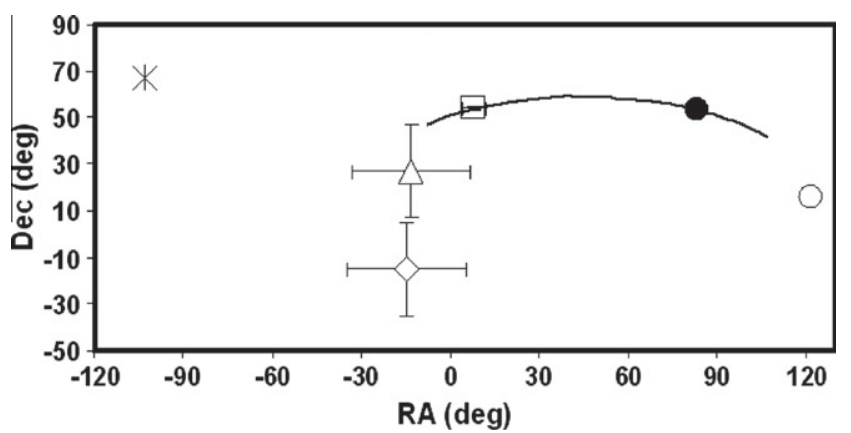

Fig. 9. Estimates of the direction of $\mathbf{M}$. The direction found in this work is marked with an open rectangle. The first approximation to this direction, based on the original Thomas et al. (2012) direction of the intermediate principal axis of inertia, is marked with a filled circle. The line joining them on the sky is the locus of possible directions given the uncertainty in the latter (see text). The direction found by Knight and Schleicher (2011) is marked with an asterisk; that by Samarasinha et al. (2010) by an open diamond; that by Waniak et al. (2012) by an open circle; and a radar based direction communicated by P.A. Taylor by an open triangle.

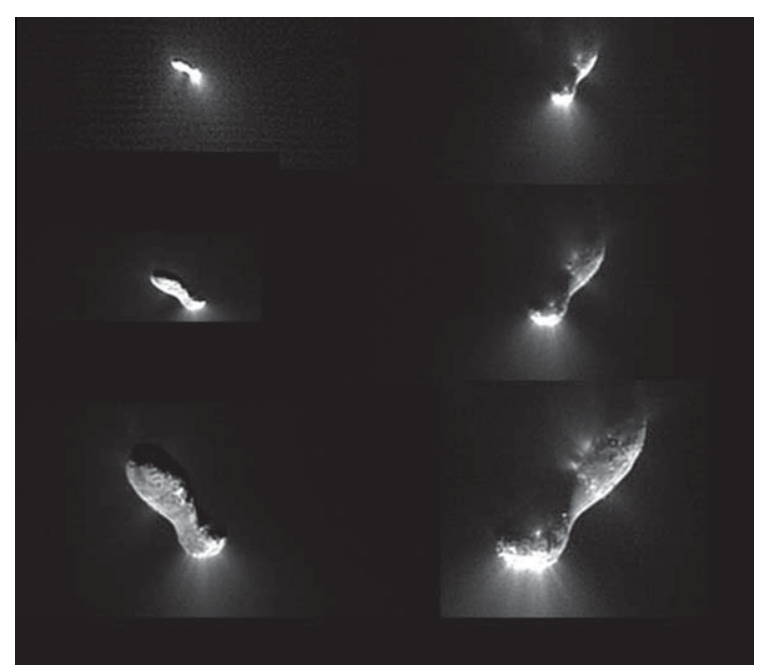

Fig. 10. Sample of three deconvolved pre-encounter HRI images (Left column) and three post-encounter images (Right column) that cover the interval Enc $-1 \mathrm{~h}$ to Enc $+1 \mathrm{~h}$. The changing position angles (PA) of the long axis, which easily be seen, in 11 such pre-encounter images were used to determine the direction of $\mathbf{M}$. Not all of the change in PA seen in this illustration is due to rotation. A fraction is due to the motion of the spacecraft relative to the comet. The post-encounter images, which have a more complex terminator structure, were not measured for PA but were used to check the result (Fig. 13).

percent during a complete cycle. The direction of $\mathbf{M}$ on the sky (assumed constant in this study) was found to be (RA, Dec; $\mathrm{J} 2000)=8 \pm 4^{\circ},+54 \pm 1^{\circ}$; however, the direction of the intermediate principal axis of inertia at closest approach estimated by Thomas et al. (2012) had to be adjusted by $43^{\circ}$ along its arc of uncertainty to $(\mathrm{RA}$, Dec; J2000 $)=301.8^{\circ},-16.5^{\circ}$ to satisfy observations of the projected long axis during the encounter.

The spin state was found to evolve during the encounter with the magnitudes of $\mathbf{M}$ and $E$ slowly decreasing through perihelion passage at rates of $-4.4 \times 10^{-7} \mathrm{~m}^{2} / \mathrm{s}^{2}$ and $4.0 \times 10^{-11} \mathrm{~m}^{2} / \mathrm{s}^{3}$ (per unit mass of the nucleus), respectively. However, even as the total energy decreased, the degree of excitation in the motion increased (Fig. 6). The average angle between the long axis and $\mathbf{M}$ decreased and as the average circulation period lengthened the average roll period decreased. To better illustrate the variability of the spin state we show in Fig. 14 predictions of the changing space direction of the instantaneous spin vector and, in Fig. 15, the changing instantaneous spin period.
The large displacement of the direction of the intermediate axis from the estimate by Thomas is perhaps not surprising given the near axial symmetry of the nucleus (in true axial symmetry there is no preferred direction for this axis) and possible imperfections in the shape model. It may indicate that some level of inhomgeneity exists in the nucleus interior, but this possibility is hard to quantify other than to say that it would be small.

In their analysis of the evolution of comet nucleus rotation, Neishtadt et al. (2002) find that axially symmetric comet nuclei with only a few active areas should tend to gain angular momentum, and align $\mathbf{M}$ with the direction toward perihelion. In the case of $103 \mathrm{P}$, a comet nucleus with similar properties, $\mathbf{M}$ is found to be some $46^{\circ}$ from the direction of perihelion, It is also losing rotational angular momentum.

\subsection{Relationship to other observations}

The somewhat complicated spin state described above clearly requires confirmation from observations other than those from which it was derived. These confirmations come from independent Earth-based observations, and we have already noted that the three $18.4 \mathrm{~h}$ cycles seen in several ground-based measurements are already built-into our model with the choice of $P_{\psi}(0)=26.72 \mathrm{~h}$. The long axis of the nucleus circulates three times for every two roll periods to bring the nucleus back to essentially the same aspect with respect to the Sun.

The secular evolution of the circulation period found in our spin state throughout the encounter is also found in ground-based observations. This confirmation is shown in Fig. 16 where the present results embed well with the ground-based record.

It is in the direction of $\mathbf{M}$ where there is a considerable divergence of opinion among ground-based studies. However, we note that Knight and Schleicher (2011) found that " ...one of the strongest constraints on the nominal rotation axis (i.e., the total angular momentum vector) is the side-on, corkscrew-like appearance of both the northern and southern features in early November. Not only do these features provide the best determined position angle through-out the apparition, yielding a great circle of deprojected pole solutions, but there is also the least amount of overlap between the two features, directly implying that we are observing from close to the comet's equator." In their Fig. 1, the image taken at UT 2010 Nov. 11 09:39 shows this configuration very clearly. The angle between our direction for $\mathbf{M}$ and the line of sight from the Earth at this time is $94.5 \pm 2.3^{\circ}$, i.e., the two directions are essentially orthogonal and so provide strong support for our result. The radar results of Harmon et al. (2011) also provide some support, in that they find that the delay-Doppler images can be well explained by an unexcited rotation about a pole of $\mathrm{RA}=17^{\circ}$, Dec $=47^{\circ}$. Our spin state is but modestly excited $\left(\theta \sim 81^{\circ}\right.$ rather that $90^{\circ}$ ), and their assumed pole is only $9.0 \pm 1.5^{\circ}$ from our result. Similarly Harmon's observations appear to be consistent with a simple rotation state with a spin axis in the region RA, $\operatorname{Dec}=(347,+27) \pm 20^{\circ}$ (P.A. Taylor, Personal communication), which partially overlaps our result. We conclude that Earth-based imaging and radar are consistent with our derived pole and therefore provide a considerable degree of confidence in it.

\subsection{Torques}

A basic assumption in this study is that the direction of $\mathbf{M}$ can be assumed fixed during the encounter. It is obviously essential to check this assumption a posteriori using the measured secular rate of change in the magnitude of $\mathbf{M}$ through the encounter, i.e., using $\mathrm{d} \mathbf{M} / \mathrm{d} t=-4.39 \times 10^{-7} \mathrm{~m}^{2} / \mathrm{s}^{2}$ per unit mass, which allows a measure of the net torque acting on the nucleus. With a total mass of $1.9-2.4 \times 10^{11} \mathrm{~kg}$ (Table 1 ), we find a net torque of 
Table 9

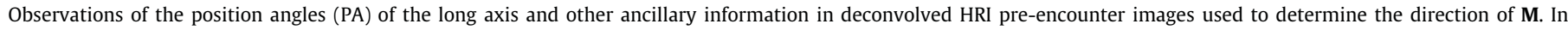
column 3, N. clock is the angle between the top of the camera frame and the projection of the North direction measured clockwise.

\begin{tabular}{|c|c|c|c|c|c|}
\hline Image & Mid-exp. JD-2455505 & N. clock $\left({ }^{\circ}\right)$ & $\mathrm{PA}\left({ }^{\circ}\right)$ & Range (km) & Phase $\left(^{\circ}\right)$ \\
\hline hv0342145836_5000005_001_rr & 0.0430539 & 73.65 & 44.75 & 42731.44 & 85.9 \\
\hline hv0342146502_5000022_001_rr & 0.0507665 & 73.64 & 49.46 & 34522.65 & 85.9 \\
\hline hv0342147067_5000065_001_rr & 0.0573033 & 73.63 & 52.08 & 27565.93 & 85.9 \\
\hline hv0342147487_5000095_001_rr & 0.0621645 & 83.93 & 53.59 & 22393.17 & 85.8 \\
\hline hv0342147802_5000118_001_rr & 0.0658104 & 83.89 & 55.06 & 18514.29 & 85.7 \\
\hline hv0342148037_5000139_001_rr & 0.0685330 & 83.85 & 56.29 & 15618.50 & 85.7 \\
\hline hv0342148201_5000152_001_rr & 0.0704317 & 83.81 & 56.11 & 13599.54 & 85.6 \\
\hline hv0342148351_5002000_001_rr & 0.0721674 & 83.75 & 57.39 & 11754.73 & 85.5 \\
\hline hv0342148501_5002015_001_rr & 0.0738996 & 83.70 & 58.26 & 9914.60 & 85.4 \\
\hline hv0342148663_5002028_001_rr & 0.0757790 & 83.62 & 59.76 & 7920.06 & 85.2 \\
\hline hv0342148790_5002044_001_rr & 0.0772484 & 83.53 & 60.76 & 6363.44 & 85.0 \\
\hline
\end{tabular}

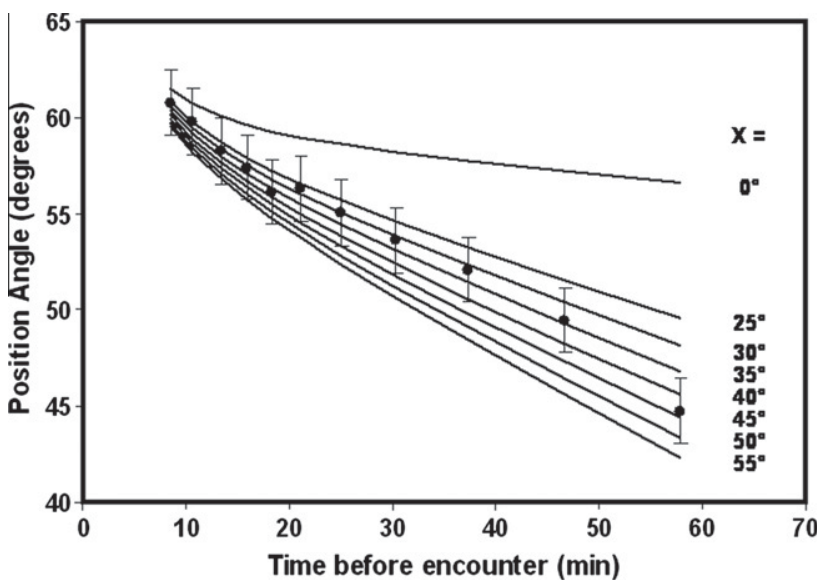

Fig. 11. Plot of the PA's (filled circles) measured on 11 pre-encounter deconvolved HRI images as a function of time from encounter. The curves represent the predicted run of PA based on directions of $\mathbf{M}$ that correspond to assumed directions of the intermediate principal axis of inertia that differ by $X^{\circ}$ from that found by Thomas et al. (2012). These directions fall on an arc of uncertainty that is normal to the direction of the long axis (see text). The coordinates assumed for $\mathbf{M}$ fall on the arc (line) shown in Fig. 9. The line marked $X=0^{\circ}$, which is well displaced from the observed points corresponds to the intermediate principal axis as found by Thomas et al. (2012). The curve for $X=43^{\circ}$ provides the best accounting of the observations as indicated in Fig. 12. For this curve the direction of the intermediate principal axis of inertia is RA, Dec $=302^{\circ},-16.5^{\circ}$.

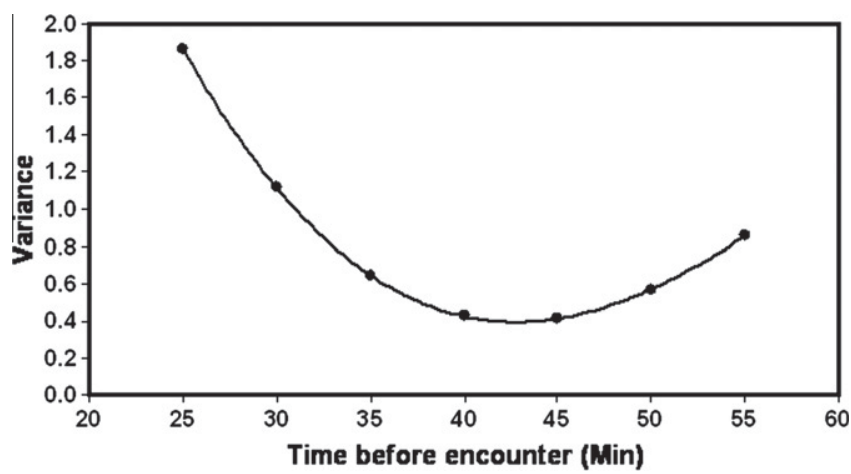

Fig. 12. Dependence of variance (between model and observed PAs) on $X$. The minimum falls at $X=43^{\circ}$.

$\sim 9.5 \times 10^{4} \mathrm{~kg} \mathrm{~m}^{2} / \mathrm{s}^{2}$ was operating near perihelion. We can get a rough estimate of the upper limit to the precession rate for $\mathbf{M}$ by imagining that a torque of this magnitude is entirely applied orthogonally to $\mathbf{M}$. Under these circumstances the precession rate

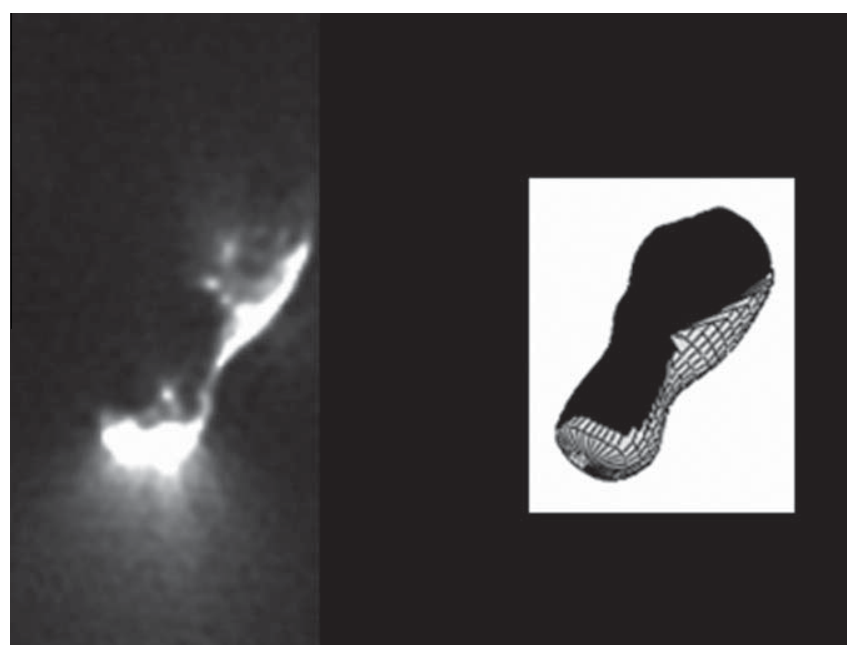

Fig. 13. Comparison of the predicted model orientation and terminator with the last post-encounter deconvolved HRI image (hv0342150580-5007045_001_rr; midexposure at JD 2455505.09796810 or $\mathrm{E}+21.29 \mathrm{~min}$ ) for our best estimate of the spin state. The image size has been adjusted but not the orientation in the image frame.

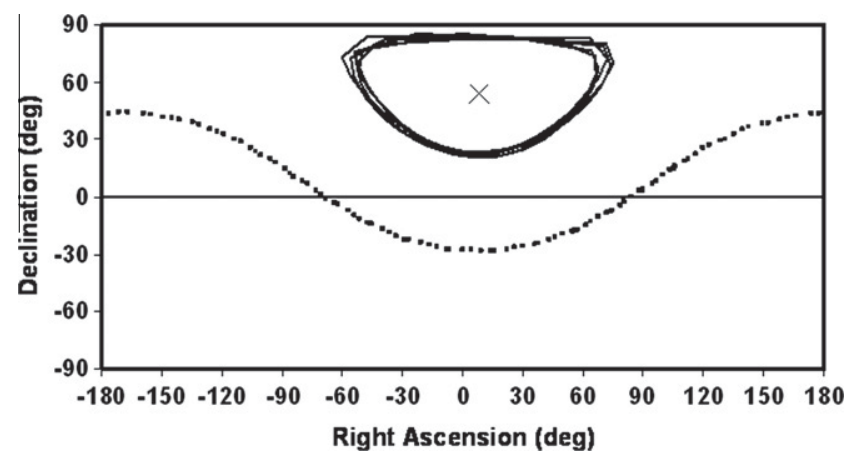

Fig. 14. Sky tracks of the long axis (dotted line) and instantaneous spin vector (continuous line) over $100 \mathrm{~h}$ centered on the time of closest approach. The cross denotes our best estimate of the direction of M. Note that the spin vector does not follow a closed curve on the sky.

is $\sim$ Torque $/ \mathbf{M}=1.5 \times 10^{-8}$ radians/s suggesting a maximum displacement of $\sim 6^{\circ}$ throughout the $81.6 \mathrm{~d}$ of the encounter. This upper limit is close to the estimated uncertainty in our determination of the direction of $\mathbf{M}$ giving some confidence in our original assumption that the direction of $\mathbf{M}$ can be considered fixed during the encounter. 


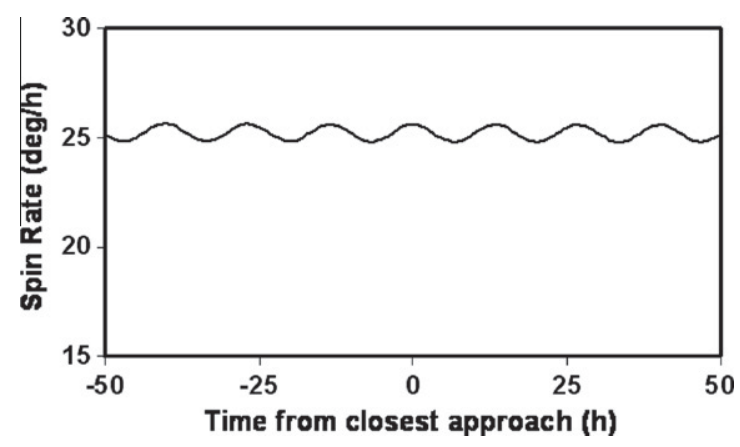

Fig. 15. The variability of the instantaneous spin rate over $100 \mathrm{~h}$ centered on the time of closest approach.

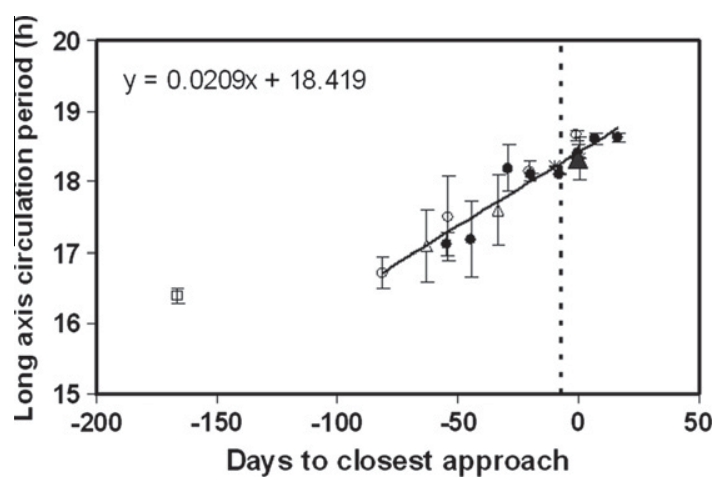

Fig. 16. Observed values of $P_{\bar{\phi}}$ (the period of circulation of the long axis around $\mathbf{M}$ ) as a function of time. DIXI results are shown as filled circles. Other observations are open circles - Knight and Schleicher (2011); open triangles - Samarasinha et al. (2011); open square - Meech et al. (2009); asterisk - Harmon et al. (2011); filled triangle - Drahus et al. (2011): cross - Waniak et al. (2012). Perihelion is marked with a vertical dotted line. The trendline is fitted, unweighted, to all of the observations except those of Meech et al. (2009).

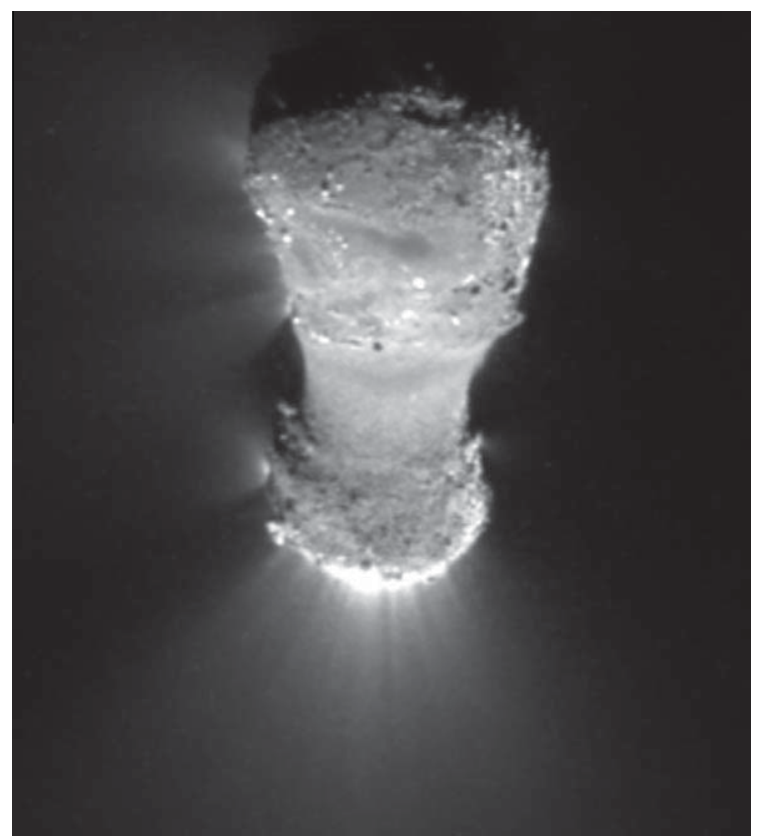

Fig. 17. High-resolution ( $8.5 \mathrm{~m} /$ pixel) MRI image (mv0342149264_5004032) was taken at JD 2455505.0827290 and shows where the evening and morning terminators meet on the big end of the nucleus. Images prior to this time are dominated by the morning terminator and those following by the evening terminator. In the image the evening part of the terminator is to the left.
Belton (2012) has already discussed the possible relationship between the net torque and the gas (essentially water vapor; $\mathrm{CO}_{2}$ has small torque potential because it is a minor constituent and its direction of outflow is roughly parallel to the long axis) production from the nucleus using a preliminary estimate of the net torque. Since that time, we have increased our estimate of the torque by $27 \%$. We will not repeat the argument in Belton (2012) except to note that with the improved estimate the surface of 103P evidently only contributes $0.8-6 \%$ of the total water vapor loss directly. The source of the bulk of the water lost by the comet apparently comes from icy particles in the coma that, to the first order, do not contribute to the net torque on the nucleus.

\subsection{Some applications}

\subsubsection{Determination of the morning and evening terminators}

Given the shortness of the encounter relative to the spin periodicities, it is not obvious from the images or the shape model alone whether the terminator that is visible in an image is the morning or evening terminator. This distinction is determined by the nature of the spin. We have used our model of the spin state to resolve this problem. Some experimentation shows that the terminator visible on approach is the morning terminator and that post-encounter exhibits the evening terminator. In images at closest approach, it is the evening terminator that is visible. We find that the transition takes place near JD 2455505.08278; images before this time show the morning terminator and those after show the evening terminator over most of the nucleus. Close to the time of transition both terminators can be seen simultaneously at the big end of the nucleus. The high-resolution MRI image (mv5004032) shown in Fig. 1 of A'Hearn et al. (2011), and reproduced here as Fig. 17, was taken at JD 2455505.0827290 and shows both terminators simultaneously in the region of the big end of the nucleus.

\subsubsection{The evolution of insolation at specific regions of the nucleus}

We have computed the variation of cosine of the angle of the Sun direction with the normal (called here the "insolation") over specific points of the nucleus to clarify our understanding of the time history of this driver for activity. In Figs. 18-20, we show the variability of the insolation with time over a 20 day period centered on the time of closest approach over the tip of the small end, a point near the origin of one of the $\mathrm{CO}_{2}$ jets at the small end $\left(20^{\circ} \mathrm{E}\right.$, $+79^{\circ}$ ), and a point near active areas seen in the evening terminator $\left(120^{\circ} \mathrm{E},-23^{\circ}\right)$ respectively. At the tip of the small end the $18.4 \mathrm{~h}$ periodicity dominates as expected. The effect of the roll period is dramatic even for the high-latitude off-axis jets and certainly for the near equatorial region. The 3-cycle periodicity can be clearly seen in the latter two curves.

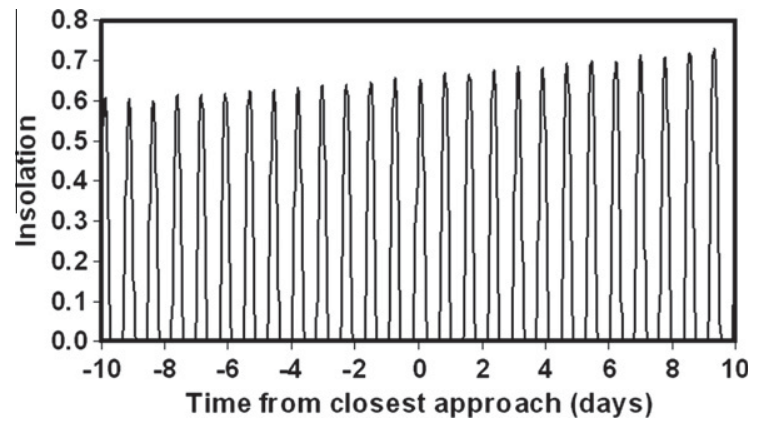

Fig. 18. Time variability of the insolation (defined as the cosine of the angle between the normal to the surface and the direction to the Sun) over the tip of the small end of the nucleus from -10 to 10 days of encounter. The $18.4 \mathrm{~h}$ periodicity is easily seen. 


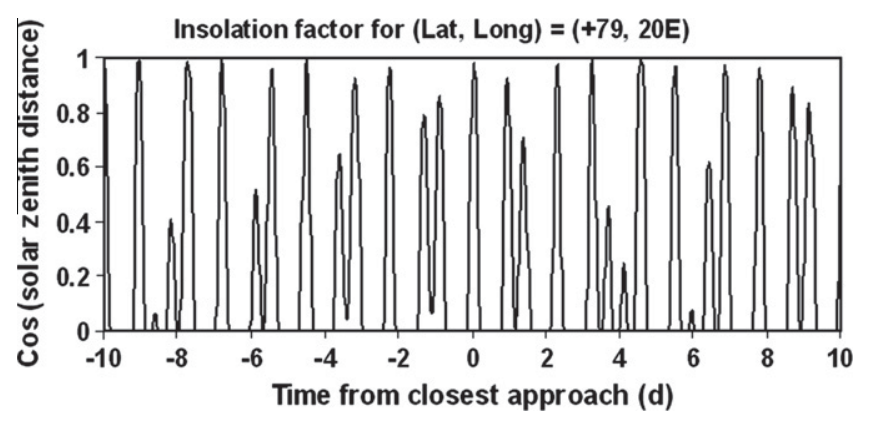

Fig. 19. Time variability of the insolation over (Lat., Long.) $=+79^{\circ}, 20^{\circ} \mathrm{E}$ a point in the middle of the cluster of $\mathrm{CO}_{2}$ jets seen near the small end of the nucleus -10 to 10 days of encounter. The insolation is strongly modulated by the roll period and gives rise to the 3-cycle periodicity that can easily be seen.

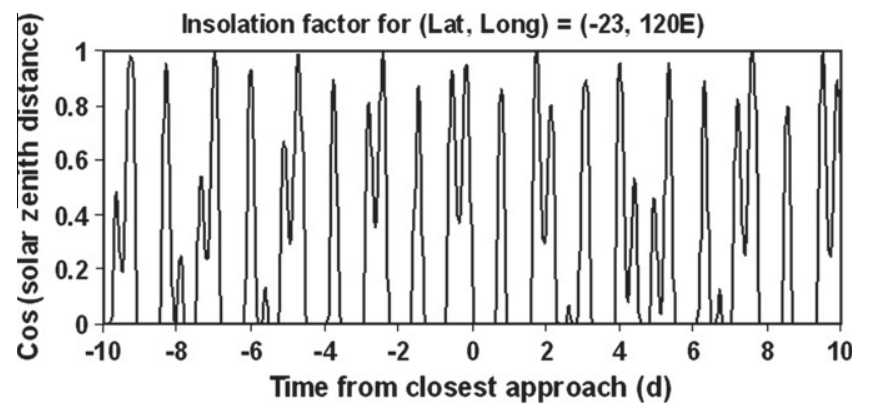

Fig. 20. Time variability of the insolation over $\left(-23^{\circ}, 120^{\circ} \mathrm{E}\right)$ a region near the strong jets seen on the evening terminator from -10 to 10 days of encounter.

\subsubsection{The source of water vapor plume}

One of the remarkable results of the DIXI mission (A'Hearn et al. (2011); see also Feaga et al., 2011) was the discovery of a plume of water vapor emanating from the region of the waist of the nucleus. Fig. 21 shows the orientation of the nucleus and terminator in a direct comparison with A'Hearn et al.'s (2011) original rendering of the water vapor data. At the time these observations were made our model predicts that there were two sub-solar regions (defined as regions with solar zenith angle $<24^{\circ}$ ) on the nucleus (because of its peculiar shape), one at the waist slightly toward the big end, and the other near the tip of the small end of the nucleus. We have included in the figure eight normals to the surface, four each from the boundaries that define each region and, as can be seen, one of the sub-solar regions, that near the waist, appears to be related to the source of the water vapor; however, the plume does not seem to propagate along the direction of the normals to the surface as might be expected if the plume issued as a jet from a vent in the surface. The normals to the surface are offset by $\sim 27^{\circ}$. The direction of the plume appears to be closer to a line that is orthogonal to the long axis and that passes through the sub-solar point (dotted line in Fig. 21), however, even this direction still diverges by $\sim 15^{\circ}$. A possible explanation for this is that the direction of the plume is under the control of the local gravitational field, since Thomas et al. (2012) have shown that gravitational potential surfaces along a meridian flattens out above the waist region for bulk densities that are in the expected range for comets. The plume may simply be warm water vapor released in relative abundance in the sub-solar region that is buoyant in the relatively dense, i.e., collisional, ambient atmosphere expected in the innermost coma and simply convects upwards.

The other peculiarity that we would note is the lack of water vapor emanating from the sub-solar region at the small end (although, according to A'Hearn et al. (2011), there is copious emis-

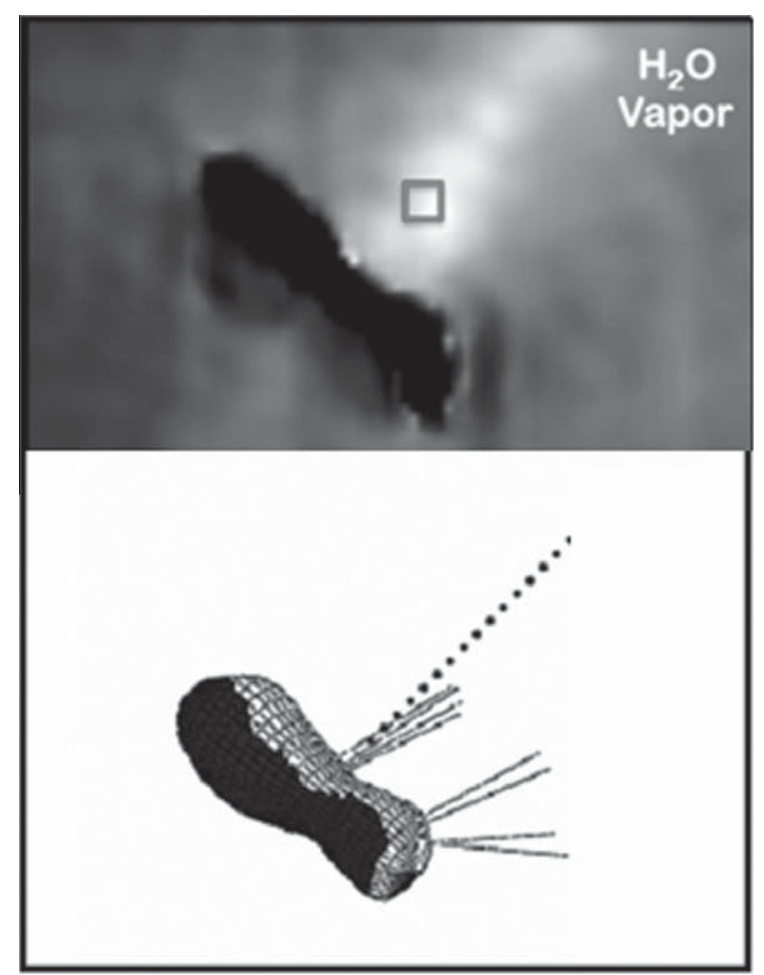

Fig. 21. Comparison of the water plume found emanating from the waist of $103 \mathrm{P} /$ Hartley 2 by A'Hearn et al. (2011) with the predicted orientation of the nucleus and terminator. The prediction is for JD 2455505.0882922 in the middle of the IR scan at $\mathrm{E}+7 \mathrm{~min}$. Also shown are normals from points of the surface that bound the two sub-solar regions (defined as regions with solar zenith angle $<24^{\circ}$ ) that existed on the surface at the time. The base the water vapor plume is consistent with its source being the sub-solar region near the waist. However, we note that the direction of the axis of the plume is not consistent with the directions of the normals to the surface, but is offset by $\sim 27^{\circ}$. It is, however, in much better accord with the direction of the normal to the long axis that passes through the sub-solar point (dotted line). This direction would be the direction of gravity if the gravitational potential is flat along a meridian as suggested in Thomas et al. (2012). The rectangle denotes the footprint of the IR spectrometer pixel. Note that the orientation of the nucleus in these images is not consistent with other images shown in this paper, which are orientated as in the original camera frame. In this figure the nucleus has been rotated anti-clockwise by $90^{\circ}$ to match the convention used in A'Hearn et al. (2011) that puts the direction of the Sun approximately to the right.

sion of water ice and $\mathrm{CO}_{2}$ from this region). It is beyond the scope of this paper to discuss the details of processes in the inner coma, nevertheless we would note that there could be a temperature effect at work here with the sub-solar region at the waist being considerably hotter than the region at the small end of the nucleus, which may be cooled by localized vaporization of $\mathrm{CO}_{2}$. Some indication of this cooling is seen in the effective temperature maps of the nucleus in Groussin et al. (2012).

\section{Acknowledgments}

This work was supported by NASA Grant NNX07AG24G under the PMDAP program and Contract NNM07AA99C from the Discovery and New Frontiers Program Office, both to the University of Maryland and sub-contracted to Belton Space Exploration Initiatives, LLC, through a Memorandum of Agreement with the National Optical Astronomy Observatories. A portion of this research was carried out at the Jet Propulsion Laboratory, California Institute of Technology, under a contract with the National Aeronautics and Space Administration. The principal author thanks the Principal Investigators Michael F. A'Hearn and Jian-Yang Li for their continuing support. We also would like to acknowledge the 
valuable assistance of Dr. D. Bockelée-Morvan in locating an error in our longitude definitions.

\section{Appendix A. Supplementary material}

Supplementary data associated with this article can be found, in the online version, at http://dx.doi.org/10.1016/j.icarus.2012. 06.037 .

\section{References}

A'Hearn, M.F., Millis, R.L., Schleicher, D.G., Osip, D.J., Birch, P.V., 1995. The ensemble properties of comets: Results from narrow-band photometry of 85 comets, 1976-1992. Icarus 118, 223-270.

A'Hearn, M.F. et al., 2011. EPOXI at Comet Hartley 2. Science 332, 1369-1400.

Ames, J.S., Murnaghan, F.D., 1929. Theoretical Mechanics. Ginn \& Co., Boston.

Belton, M.J.S., 2012. Cometary evolution and cryo-volcanism. Canadian J. Phys., in press (Hunten Special Issue).

Belton, M.J.S. et al., 2011a. The spin of 103P/Hartley 2 and its evolution during the EPOXI/DIXI encounter. Lunar. Planet Sci. 42. Abstract 1607.

Belton, M.J.S. et al., 2011b. Stardust-NExT, Deep Impact, and the accelerating spin of 9P/Tempel 1. Icarus 213, 345-368.

Bodewits, D., Farnham, T., Kelley, M.S., and The DIXI team, 2012. Gas and dust in the coma around Hartley 2. Icarus, in review.

Drahus, M. et al., 2011. Rotation state of Comet 103P/Hartley 2 from radio spectroscopy at $1 \mathrm{~mm}$. Astrophys. J. 734, L4-1-6.

Feaga, L.M. et al., 2011. Heterogeneity of Comet 103P/Hartley 2's gaseous coma. Lunar Planet. Sci. 42. Abstract 2461.

Groussin, O. et al., 2012. In-situ thermal observations of the nuclei of Comets 103P Hartley and 9P/Tempel 1. Icarus, in review.

Harmon, J.K. et al., 2010. IAU Circ. 91790.1.
Harmon, J.K., Nolan, M.C., Howell, E.S., Giorgini, J.D., Taylor, P.A., 2011. Radar observations of Comet 103P/Hartley. Astrophys. J. 734, L2-1-4.

Jehin, E., Manfroid, J., Hutsémekers, D., Guillon, M., Magain, P., 2010. CBET. 2589.1 Knight, M., Schleicher, D.G., 2010. IAU Circ. 9175, 2.

Knight, M.M., Schleicher, D.G., 2011. CN morphology studies of Comet 103P/Hartley 2. Astron. J. 141, 183.

Lindler, D.J. et al., 2012. Interpretation of results of deconvolved images from Deep Impact spacecraft High Resolution Imager. Icarus, in review.

Lisse, C.M. et al., 2009. Spitzer Space Telescope observations of the nucleus of Comet 103P/Hartley 2. Publ. Astron. Soc. Pacific 121, 968-975.

Meech, K.J. et al., 2009. Characterization of the nucleus of 103P/Hartley 2 for the EPOXI mission. Bull. Am. Astron. Soc. 41, 1029.

Meech, K.J. et al., 2011. EPOXI Comet 103P/Hartley 2 observations from a worldwide campaign. Astrophys. J. 734, L1-1-9.

Neishtadt, A.I., Scheeres, D.J., Sidorenko, V.V., Vasilev, A.A., 2002. Evolution of comet nucleus rotation. Icarus 157, 205-218.

Samarasinha, N.H., A'Hearn, M.J., 1991. Observational and dynamic constraints on the rotation of Comet P/Halley. Icarus 93, 194-225.

Samarasinha, N.H., Mueller, B.E.A., Belton, M.J.S., Jorda, L., 2004. Rotation of cometary nuclei. In: Festou, M.C., Keller, H.U., Weaver, H.A. (Eds.), Comets II University of Arizona Press, Tucson, pp. 281-299.

Samarasinha, N.H., Mueller, B.E.A., A'Hearn, M.F., Farnham, T.L. 2010. IAU Circ. 9178

Samarasinha, N.H., Mueller, B.E.A., A'Hearn, M.F., Farnham, T.L., Gersch, A., 2011. Rotation of Comet 103P/Hartley from structures in the coma. Astrophys. J. 734 L3-1-6.

Schwarzenberg-Czerny, A., 1991. Accuracy of period determination. Mon. Not. R. Astron. Soc. 253, 198-206.

Schwarzenberg-Czerny, A., 1996. Fast and statistically optimal period search in uneven sampled observations. Astrophys. J. 460, L107-L110.

Thomas, P.C. et al., 2012. Shape and geology of Comet 103P/Hartley 2. Icarus 222 550-558.

Waniak, W., Borisov, G., Drahus, M., Bonev, T., 2012. Rotation-stimulated structures in the $\mathrm{CN}$ and $\mathrm{C} 3$ comae of Comet 103P/Hartley 2 around the EPOXI encounter. Astron. Astrophys. 543, A32-A39. 